Exp Brain Res (1990) 81:1-17

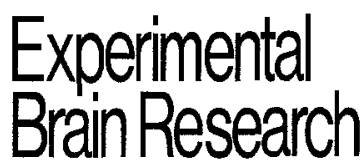

(C) Springer-Verlag 1990

\title{
The topology of the thalamo-cortical projections in the marmoset monkey (Callithrix jacchus)
}

\author{
W. Brysch, I. Brysch, O.D. Creutzfeldt, R. Schlingensiepen, and K.-H. Schlingensiepen \\ Abteilung Neurobiologie, Max-Planck-Institut für Biophysikalische Chemie, Postfach 2841, D-3400 Göttingen, \\ Federal Republic of Germany
}

Received September 11, 1989 / Accepted December 31, 1989

Summary. This paper addresses the question of a general topological principle of thalamo-cortical projections. In the lissencephalic primate brain of the common marmoset (Callithrix jacchus), large injections of horseradish peroxidase were made in various parts of the neocortex. These injections were placed in different animals and hemispheres along various caudo-rostral and mediolateral gradients. Labelled cells in the thalamus were plotted and the labelling-zones resulting from several injections along a medio-lateral and two caudo-rostral cortical vectors were drawn into semi-schematic thalamic maps. These composite maps reveal a topological organization of the whole thalamo-cortical projection. The thalamic representation of the caudo-rostral and mediolateral gradients indicate a rotation of the posterior relative to the anterior thalamus. An attempt is made to relate the organization of the thalamo-cortical projection to the development of the thalamus and the cortex. The cortex is divided into concentric zones around the sensory-motor and insular cortex. The thalamus is divided into corresponding projection zones. The topology of thalamo-cortical connections can then be regarded as a consequence of corresponding thalamic and cortical

\footnotetext{
Abbreviations: AD Nucleus anterior dorsalis thalami; AM Nucleus anterior medialis thalami; AV Nucleus anterior ventralis thalami; CeD Nucleus centralis dorsalis thalami; CeL Nucleus centralis lateralis thalami; CeM Nucleus centralis medialis thalami; CeMe Centre median; GLD Corpus geniculatum laterale dorsale; GLV Corpus geniculatum laterale ventrale; GM Corpus geniculatum mediale; LD Nucleus lateralis dorsalis thalami; Li Nucleus limitans thalami; LP Nucleus lateralis posterior thalami; MD Nucleus medialis dorsalis thalami; Pf Nucleus parafascicularis thalami; PvT Nucleus paraventricularis thalami; PuI Pulvinar inferior; PuIP Pulvinar inferior posterior; PuL Pulvinar lateralis; PuM Pulvinar medialis; PuO Pulvinar oralis; Re Nucleus Reuniens; Sg Nucleus suprageniculatus; VA Nucleus ventralis anterior; VAMg Nucleus ventralis anterior magnocellularis; VL Nucleus ventralis lateralis; VP Nucleus ventralis posterior; VPL Nucleus ventralis posterior lateralis; VPM Nucleus ventralis posterior medialis
}

Offprint requests to: O.D. Creutzfeldt (address see above) growth gradients. This is not only consistent with the general thalamo-cortical topology and the inversion of maps from thalamus to cortex, but also explains the continuity and overlap of thalamic projection zones in the pulvinar to widely separated cortical areas as the parietal, temporal and frontal association cortex.

Key words: Thalamo-cortical connections - Topology Development - Specific and intralaminar thalamic nuclei - Primate

\section{Introduction}

All neocortical areas receive afferent fibres from a circumscribed region or nucleus of the thalamus (see for example Walker 1938; Jones 1985). These thalamo-cortical projections are organized in a topological manner: Neighbouring thalamic regions project to neighbouring cortical points, with various degrees of scatter and mutual overlap. This is most obvious for the various organotopic maps in the thalamus and cortex but it can also be demonstrated for those thalamo-cortical projections, which do not show obvious somatotopy. Thus the projections from the posterior thalamus to the occipital and temporo-occipital cortex are well documented to be topologically organized (Jones 1974; Berson and Graybiel 1978; Creutzfeldt 1985). The same applies for the thalamic projections to the frontal and prefrontal primate cortex (Kievit and Kuypers 1977; Goldman-Rakic and Porrino 1985).

On the other hand, it is obvious that the topological vectors of neighbourhood progression vary considerably from rostral to caudal parts of the thalamo-cortical projection system in that thalamic maps are differently rotated relative to the cortical ones. Thus, for example, the somatotopic progression (from head to hind legs) runs from medio-ventral to latero-dorsal in the somato- 
sensory and motor thalamic nuclei (Hassler et al. 1979), but from lateral to medial in the sensory-motor cortex. A similar topographic organization of thalamo-cortical projections throughout the thalamus has also been demonstrated to exist in aquatic mammals (Revishchin and Garey 1990).

In the prefrontal-frontal cortex on the other hand, a rostro-caudal progression roughly corresponds to a medio-lateral progression in the thalamus (Kievit and Kuypers 1977; Goldman-Rakic and Porrino 1985). In the cat's occipito-temporal cortex a medio-lateral progression from the peristriate to the temporal association cortex is matched, roughly speaking, by a latero-medial progression in the thalamus from the lateral geniculate nucleus through the pulvinar-lateralis posterior (LP) complex to LP medialis (Berson and Graybiel 1978; Creutzfeldt 1985).

In the present study we want to address the question whether a common topological rule can be defined for the mapping of thalamo-cortical projections in general. For this purpose we have chosen the common marmoset because of its essentially lissencephalic cortex but highly developed primate brain. We used relatively large injections of horse-radish-peroxidase (HRP) into various cortical areas and traced their thalamic afferents. On the basis of our experiments we propose a model of thalamocortical organisation which takes into account the phylogenetic and ontogenetic development of the thalamus as well as details of thalamo-cortical mapping.

In an accompanying study on the organisation of thalamo-cortical projections in the hamster and guinea pig, the model is further corroborated (Hoehl-Abrahano and Creutzfeldt, in preparation).

\section{Material and methods}

In 16 hemispheres of nine adult marmosets we made relatively large injections of HRP in various regions of the lateral convexity of the cortex (Figs. 1A, C and 2). For these injections the animals were anaesthetised with $\mathrm{Nembutal}^{\circledR}(30 \mathrm{mg} / \mathrm{kg}$ intraperitoneally). Additional doses were given during the operation. The animals woke up from anaesthesia not earlier than $10 \mathrm{~h}$ after the operation and were somnolent for another $8-10 \mathrm{~h}$. Their behaviour during the survival period did not indicate any painful discomfort.

After exposing the cortical surface at the respective site 0.4-0.6 $\mathrm{\mu l}$ of HRP (approximately $50 \% \mathrm{w} / \mathrm{v}$ in lysolecithin/DMSO solution) were injected, using a Hamilton syringe. The HRP solution was administered over a $10-15 \mathrm{~min}$ period. The injection needle was left in place for a further $15 \mathrm{~min}$ period to allow for better diffusion of the tracer into the tissue. After a survival time between 24 and $48 \mathrm{~h}$, the animals were perfused transcardially after lethal Nembutal anaesthesia $(90 \mathrm{mg} / \mathrm{kg})$ with $1-1.51$ of $0.9 \% \mathrm{NaCl}$ in $0.1 M$ phosphate buffer, followed by $1-21$ of fixative (1\% paraformaldehyde, $1.5 \%$ glutaraldehyde, $0.02 \% \mathrm{CaCl}_{2}$ in $0.1 \mathrm{M}$ phosphate buffer) and 11 of $10 \%$ sucrose in $0.1 M$ phosphate buffer. The brains were removed from the skull and kept at $4^{\circ} \mathrm{C}$ in a $20 \%$ sucrose solution overnight. Coronal cryostat sections at $28 \mu \mathrm{m}$ were cut. HRP was visualised by the TMB-method (Mesulam 1982) and the sections were counterstained with neutral red. The location and distribution of retrogradely labelled cells were mapped using an $\mathrm{XY}$-plotter, controlled by two linear potentiometers which were attached to the microscope stage.

\section{Cytoarchitecture and nomenclature}

Subdivisions and nomenclature of thalamic nuclei were adopted from the atlas of the marmoset brain by Stephan et al. (1980). For our semischematic map of the thalamus and for assigning labelled cells to certain nuclei we have also taken into account own observations on horizontally and coronally cut brains, which were stained for cells with cresyl-violet or silver stained for fibres in alternate sections. Where possible the cytoarchitectonic and myeloarchitectonic criteria of several authors were used to delineate borders between thalamic nuclei and subnuclei: Vogt (1909), Malone (1910), Hassler (1950), Jones (1985), Hirai and Jones (1989).

Some nuclear borders are difficult to define on cytoarchitectonic or myeloarchitectonic grounds, especially in the rostro-caudal plane. Our divisions are therefore mainly meant to aid orientation within the thalamus. Cortical areas are delineated according to Brodmann's (1909) criteria and his map of the marmoset brain (Fig. 1B).

\section{Results}

In order to show the topography of the various injection sites (Fig. 1A) and their location relative to each other, undistorted by the curvature and the sulci of the cortex, we constructed a two-dimensional topological map of the cortex which we derived from frontal sections of the brain (Fig. 1C).

We placed the various injections along rostro-caudal and medio-lateral lines on the lateral surface of the cortex. In experiment 11 two injections were placed next to each other in the suprasylvian cortex. Injection 12 involved the upper and lower lip of the Sylvian fissure (12a and $\mathrm{b}$ respectively; see Figs. $1 \mathrm{C}$ and 2 ).

The location of retrogradely labelled cells in the thalamus was transferred from the original plots into semischematic maps of frontal sections of the thalamus (see Methods). The number of HRP-labelled cells varied in the different experiments due to the size of the injec-

Fig. 1. A Lateral view of the marmoset's brain showing the localisation of the various injection sites. B Brodmann's (1909) map of the marmoset cortex. We have superimposed on this the anteroposterior (AP) ordinates. C Two-dimensional topological map of the cortex, derived from frontal sections of the brain. The circumference of coronal sections was measured at $1 \mathrm{~mm}$ intervals and drawn as straight lines. The circumferences of sections A 1-A 13 (centro-parietal cortex) start at the corpus callosum, which was drawn as a straight reference line (A 2). The circumference of the prefrontal cortex starts at the edge between basal and mesial cortex (A 3) and in the occipital region at the upper lip of the calcarine fissure (reference line A 1). The interhemispheric fissure is marked by line $\mathrm{C}$. The transition from the lateral to the basal cortex is indicated by line D. The Sylvian fissure was unfolded and as a consequence the straight coronal lines had to be bent around the depth of the Sylvian fissure. The continuation of this line onto the convexity corresponds roughly to the border between the parietal and the temporal association cortex (line B). In order to preserve topological continuity across line B the distances between adjacent AP-lines had to be increased by a factor of 2 and their length decreased accordingly by a factor of $1 / 2$. From A 20-A 13 (along A 3), AP-distances increased and diverged, and the lengths of the respective coronal circumferences were reduced accordingly 

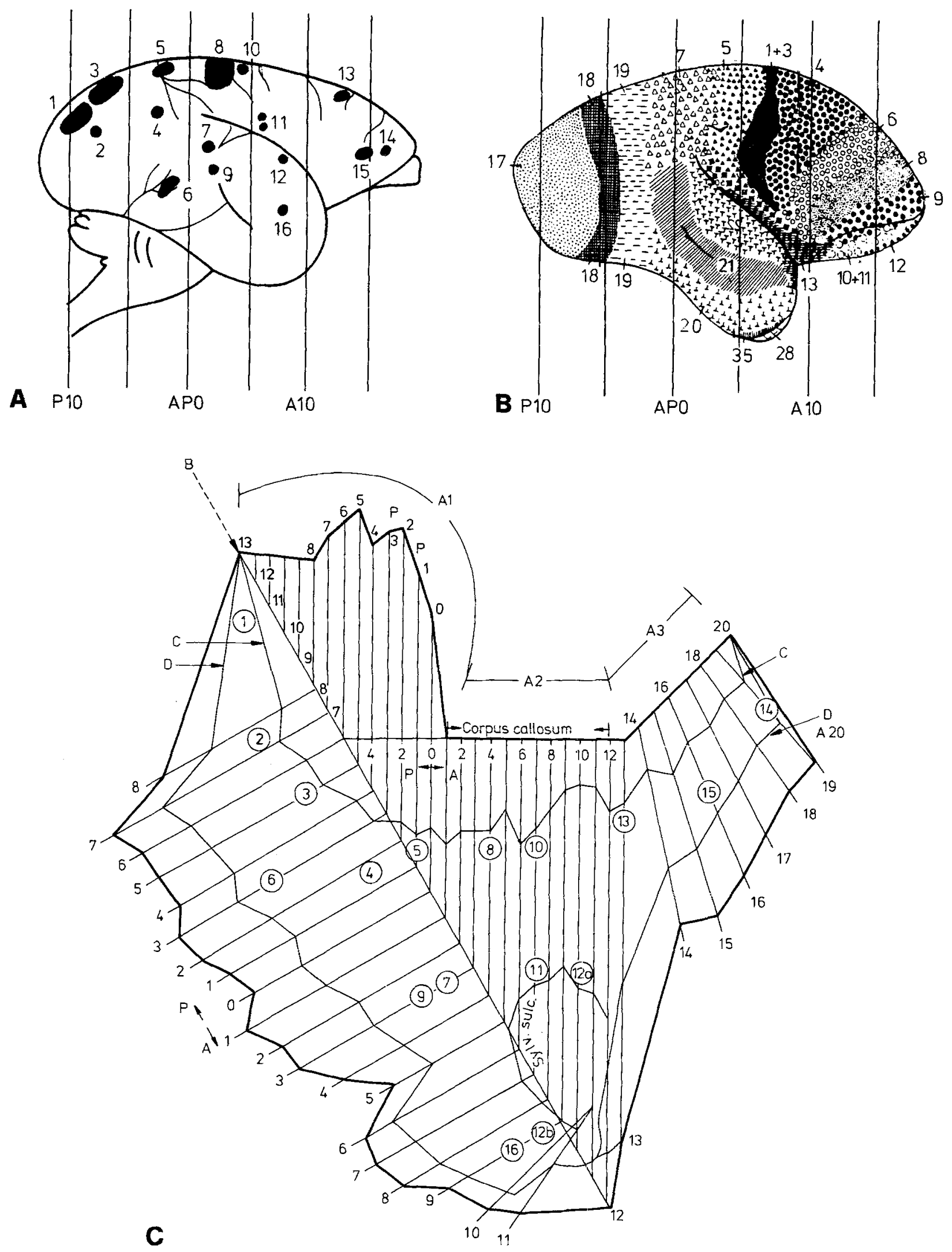

Fig. 1 


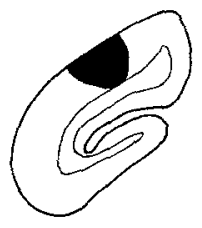

1

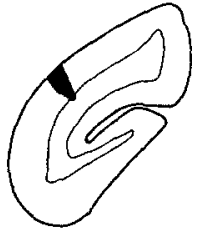

2

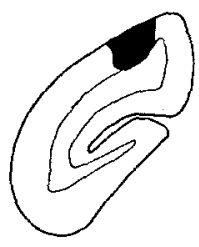

3
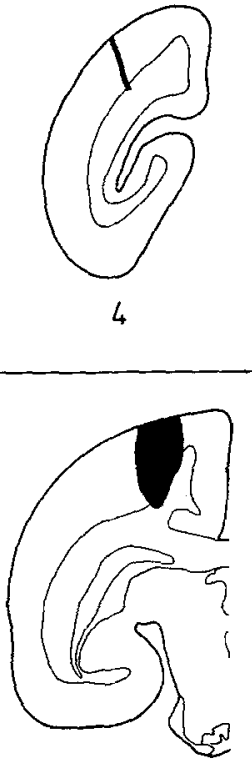

8

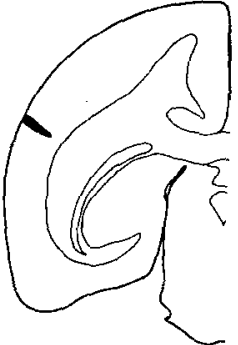

9

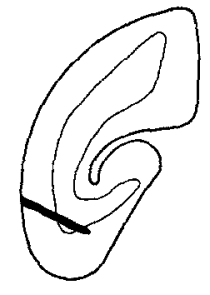

6

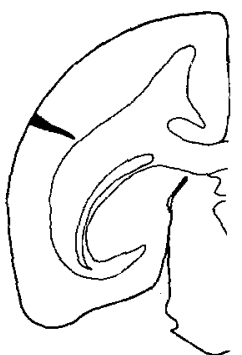

7

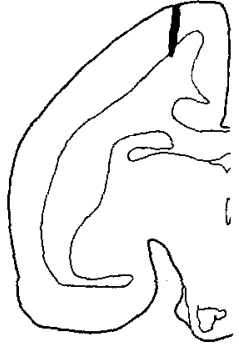

10

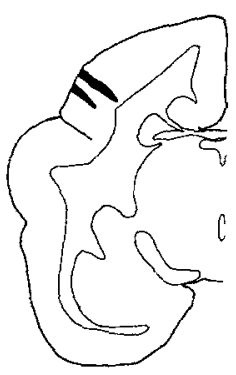

11

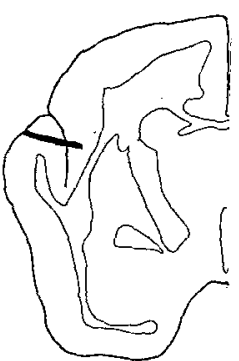

12

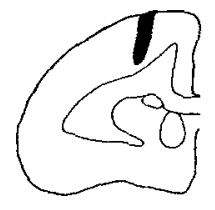

13

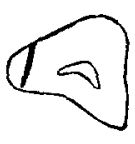

14

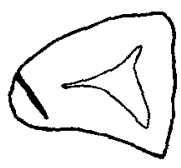

15

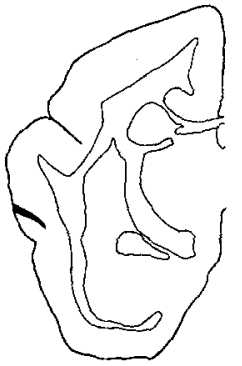

16
Fig. 2. Coronal sections through the deepest parts of the individual HRP-injections. The black marks indicate the zone of HRP-uptake according to Mesulam (1978). Experiments are numbered as in Fig. 1 tions, the exact position relative to the terminating thalamo-cortical fibres and possibly also due to the variable density of afferent fibres in various cortical regions. We will not go into the details of these variations, as we are interested only in the topographic location of the thalamic projection zones. In the plots, the number of symbols does not represent the exact number of cells in that position, but only reflects their relative density. Details of the original plots are available (Brysch 1987).

\section{Continuities and discontinuities between labelling zones in the specific and intralaminar nuclei}

Almost all cortical injections labelled thalamic neurons which were arranged in coherent clouds. These mostly stretched in various directions through several thalamic nuclei. Such continuous projection clouds were found even in regions, where a nucleus is displaced from the rest of the thalamus such as the dorsal lateral geniculate nucleus (GLD). Thus, injections 1-3 into the striate and prestriate cortex labelled cell groups in the GLD and the pulvinar which were in contact where both nuclei touch each other (open triangles in Figs. 6 and 7, plane A 3.5). Intralaminar and specific thalamic labelling fields were continuous after injections into a wide zone around the somato-sensory cortex (injections $8,10,11,12$, and 13) as specific thalamic labelling fields from these experiments cover the nuclei lying next to the intralaminar zone, namely medialis dorsalis (MD), LP, the ventral nuclei and the medio-anterior part of the pulvinar. 


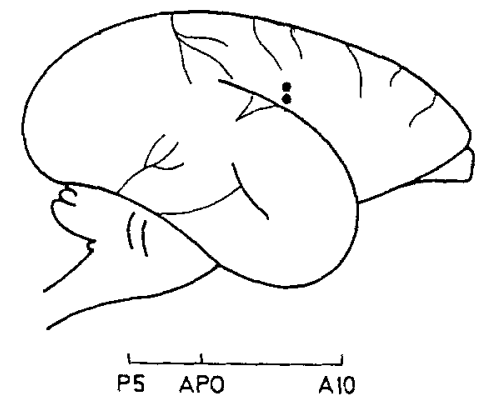

A 4.5

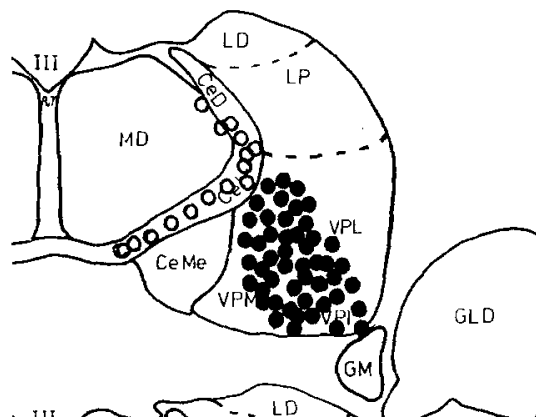

A 4

A 7

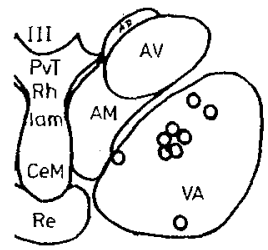

A 6.5

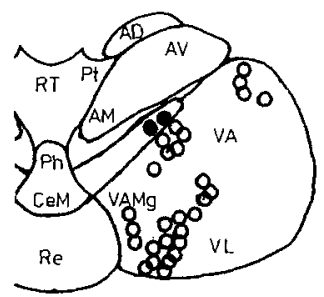

A 3.5

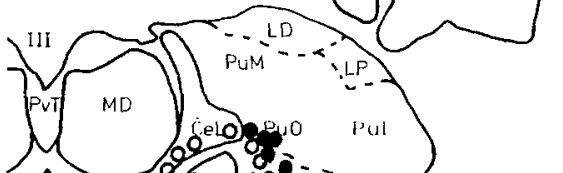

A 3

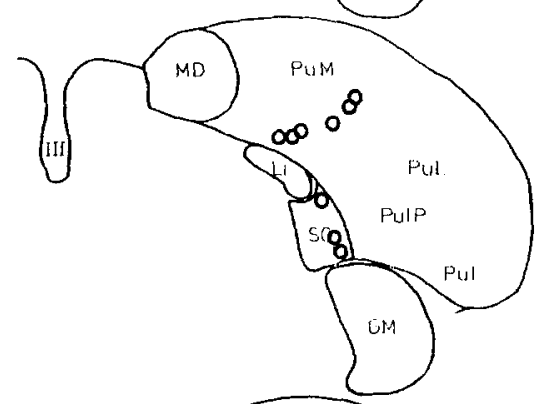

A 5.5
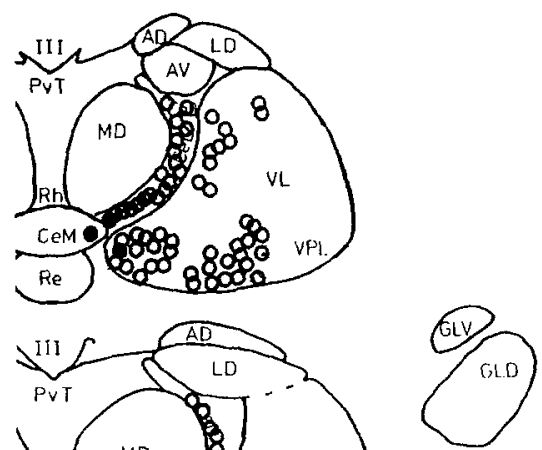

\section{A 6}
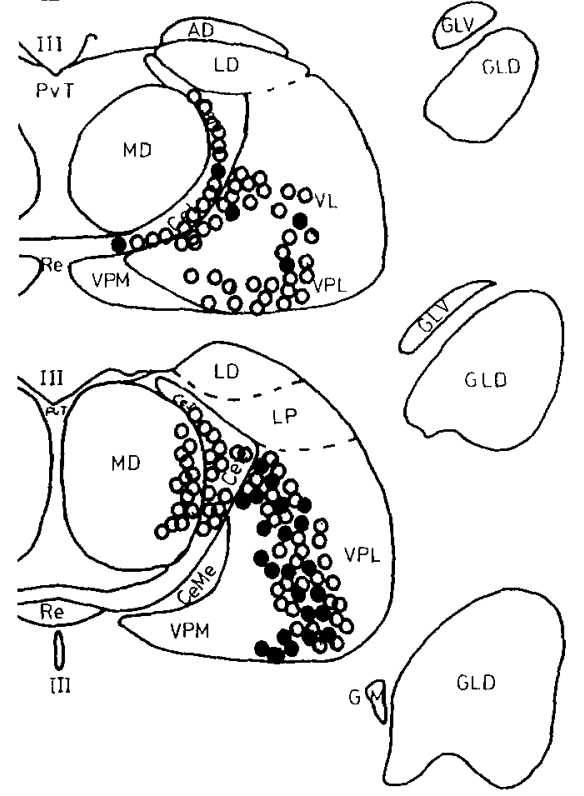

A 5

A 2.5
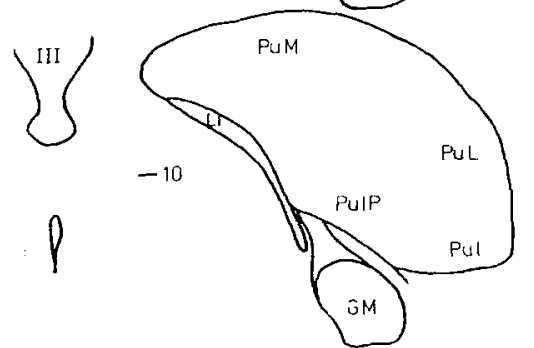

A 2
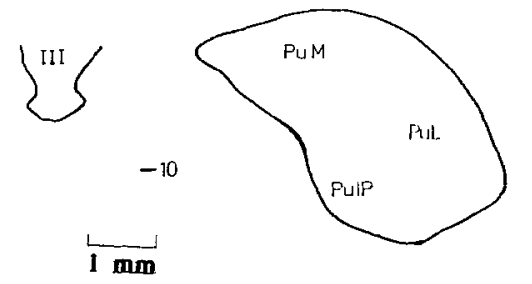

Fig. 3. Diagrammatic representation of thalamic labelling in experiment 11 , showing a continuous labelling zone covering specific and intralaminar nuclei. Filled circles represent ten, open circles one labelled cell

Examples are shown in Figs. 3 and 4: In experiment 11 (Fig. 3) two HRP injections were made in the upper lip of the Sylvian fissure, i.e. in the border region between the somato-sensory cortex and the parietal association cortex (area 5). These injections labelled a coherent field of specific thalamic projection nuclei including the medial segments of nucleus ventralis lateralis (VL) and nucleus ventralis posterior lateralis (VPL) as well as a 


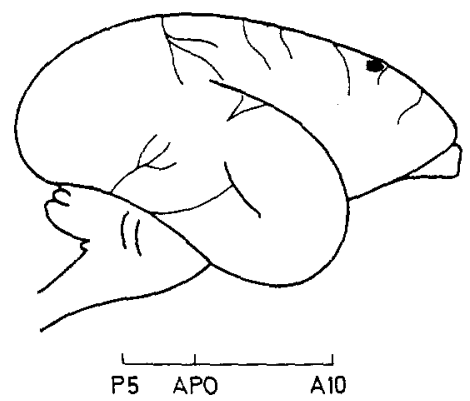

A 7

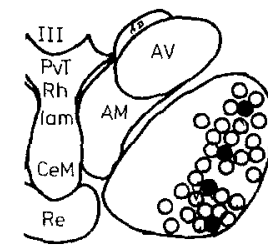

A 6.5

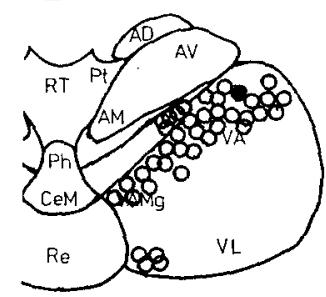

A 6
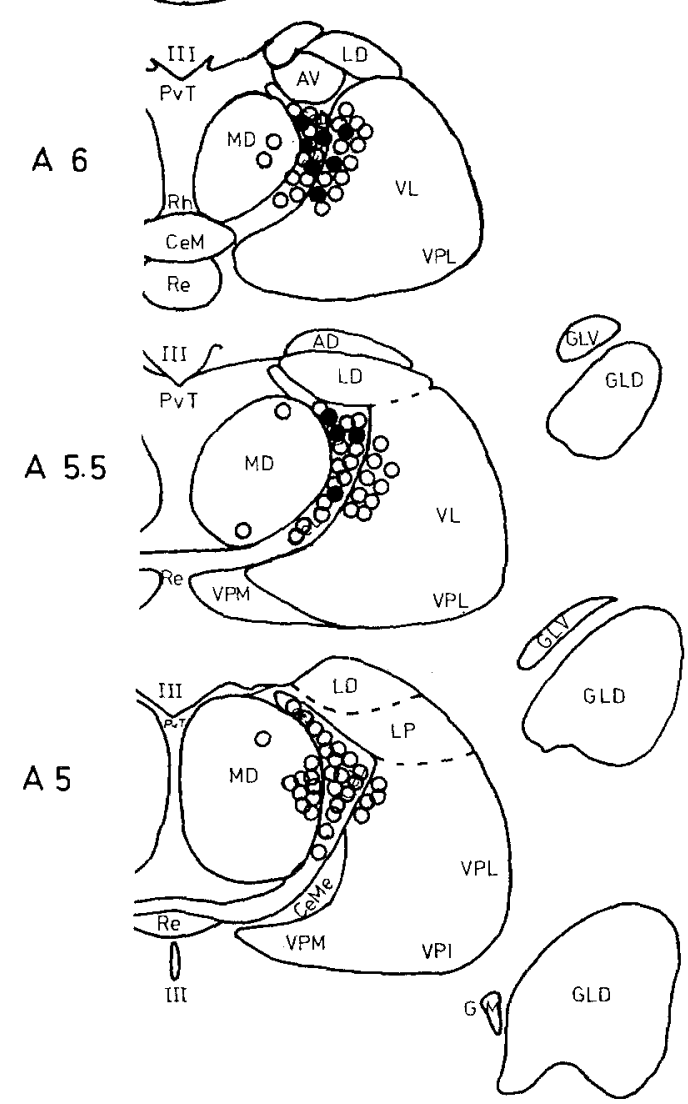

A 4.5

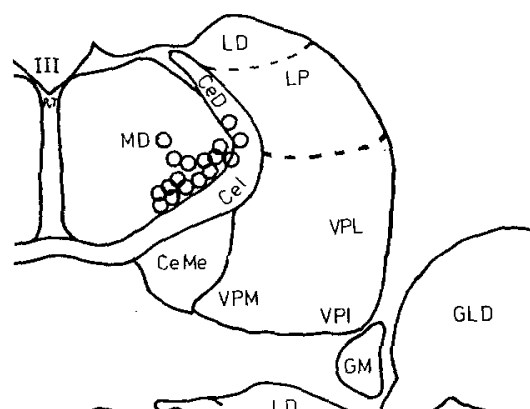

A 4

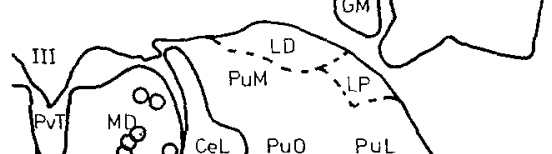

A 3.5

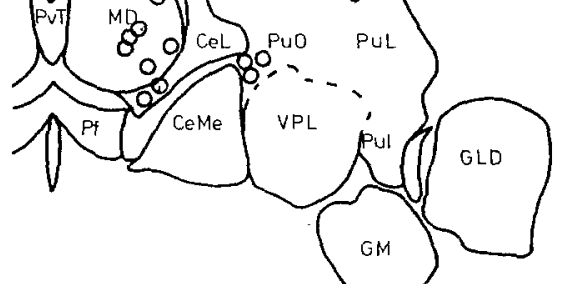

A 3

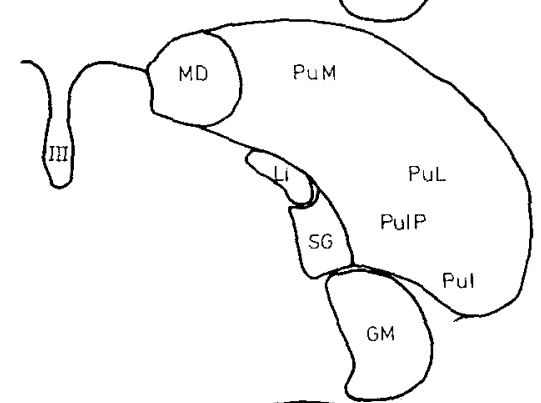

A 2.5
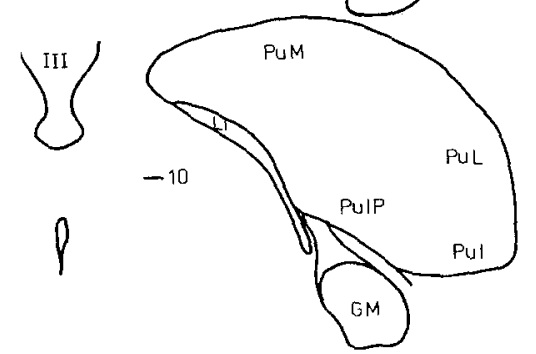

A 2
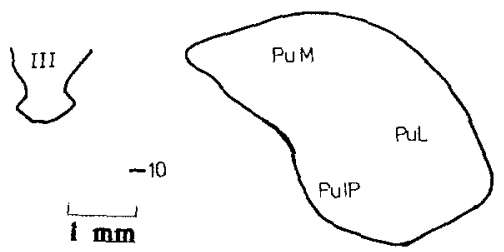

Fig. 4. Thalamic labelling following injection 13 , showing a continuous labelling zone reaching from MD through the intralaminar nuclei and VL to the medial pulvinar. Symbols as in Fig. 3 medio-basal section of the pulvinar (pulvinar oralis, $\mathrm{PuO}$ ) and the supra-geniculate nucleus (SG). This specific projection zone is continuous with the nonspecific intralaminar zone in the nucleus centralis lateralis $(\mathrm{CeL})$. In experiment 13 (Fig. 4) the prefrontal cortex was in- jected at the transition zone between area 4 and 6 . The labelling zone stretches continuously through the lateral part of MD, the medial parts of the nuclei ventralis anterior (VA), VL and $\mathrm{PuO}$ as well as the intralaminar nuclei $\mathrm{CeL}$ and centralis dorsalis $(\mathrm{CeD})$. Real discon- 


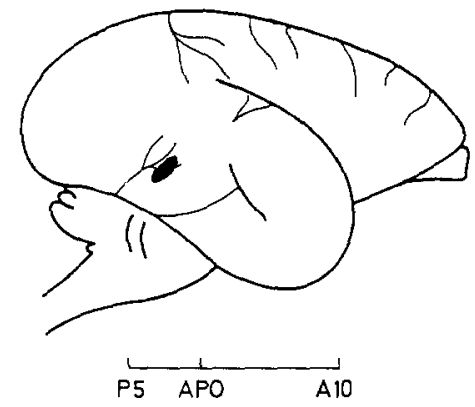

A 4.5

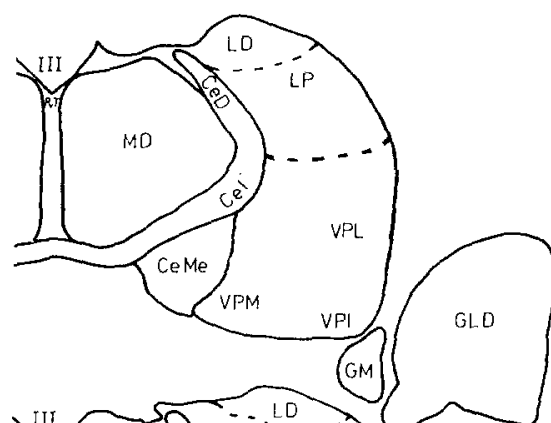

A 4

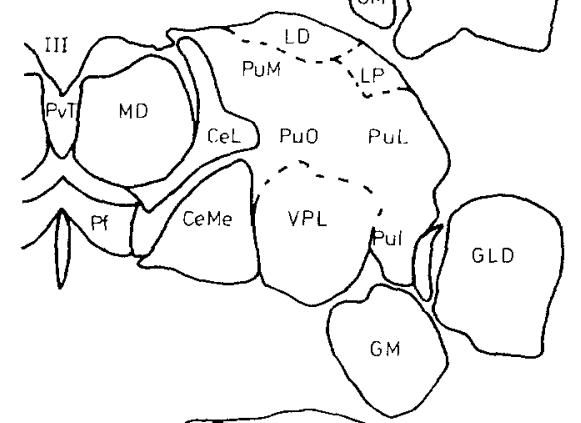

A 7

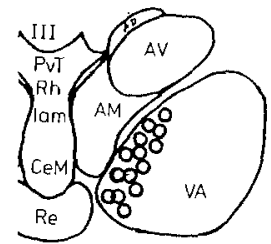

A 6.5

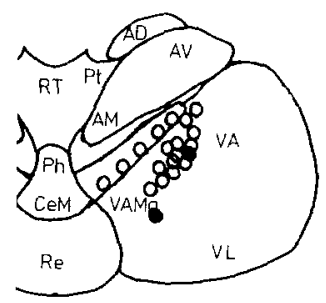

A 3.5

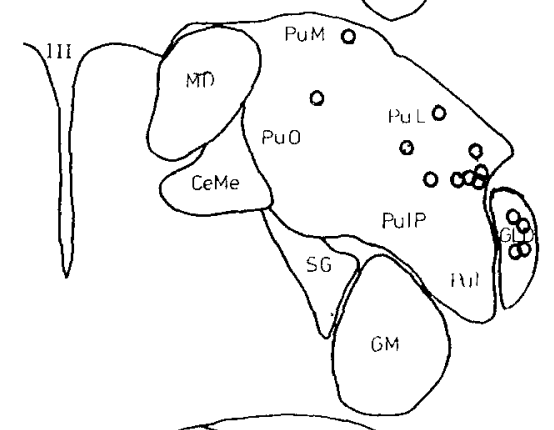

A 6
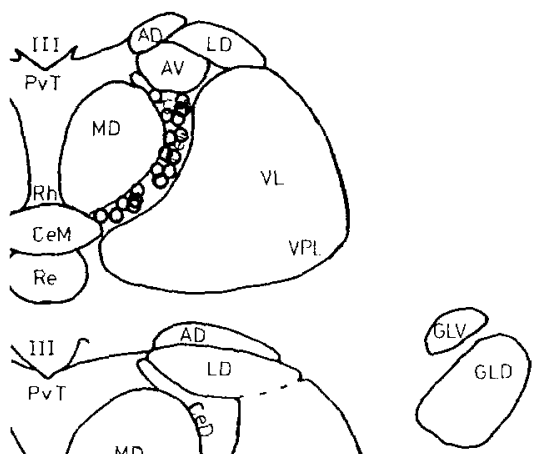

A 5.5
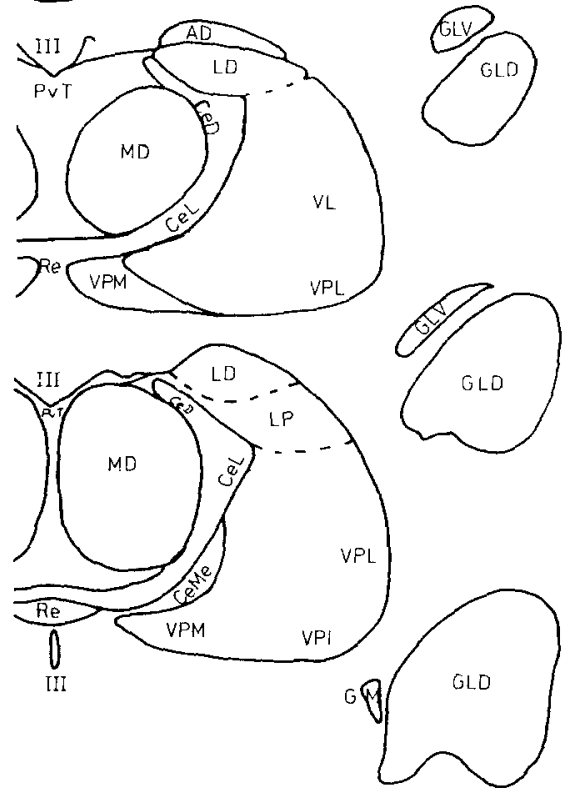

A 2.5

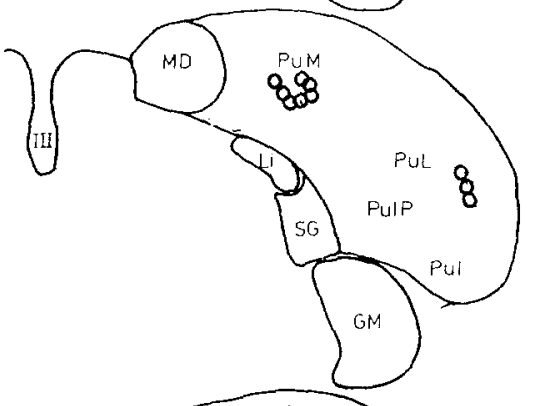

A 3
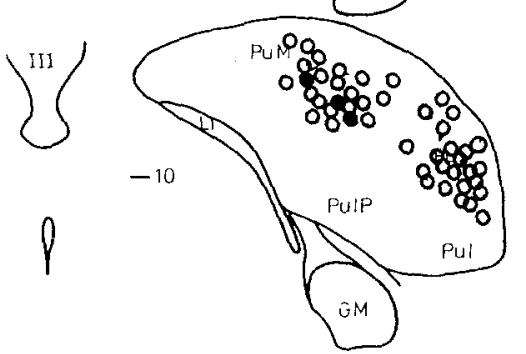

A 5

\section{A 2}
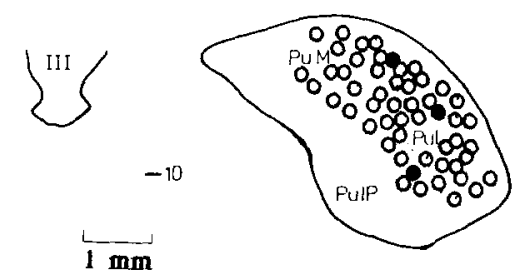

Fig. 5. Thalamic labelling in experiment 6 . Labelling in the lateral pulvinar is clearly separate from labelling in the intralaminar nuclei and VA. Symbols as in Fig. 3

tinuities between labellings in the specific and the nonspecific intralaminar nuclei were only seen where both are clearly separated from each other. This was the case in the experiments with occipital and temporo-occipital injections, which labelled cells in GLD and the postero- lateral pulvinar, i.e. parts of the thalamus not lying next to the intralaminar zone (see for example experiment 6 , Fig. 5).

In all experiments an extension of the intralaminar labelling zone into neighbouring "specific" nuclei was the 


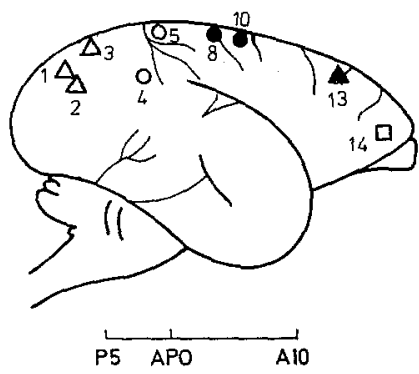

A 7

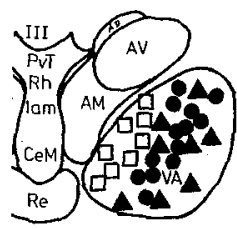

A 6.5

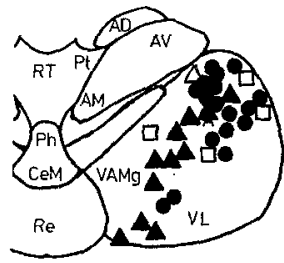

A 6

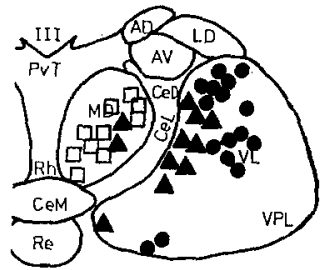

A 5.5
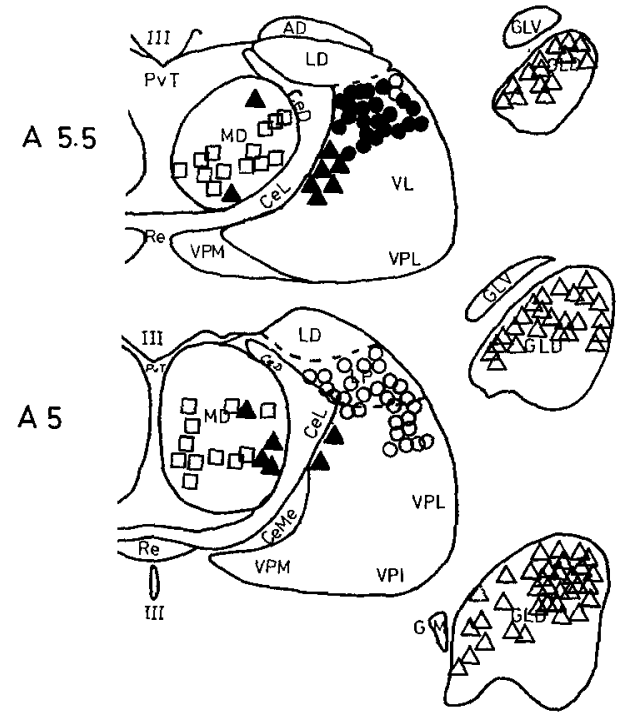

A 4.5

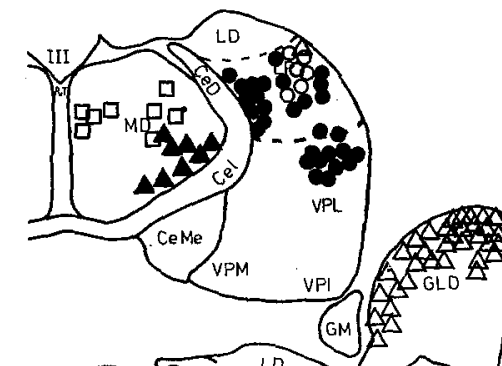

A 4

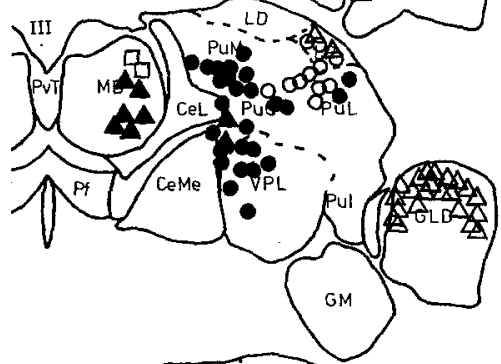

A 3.5

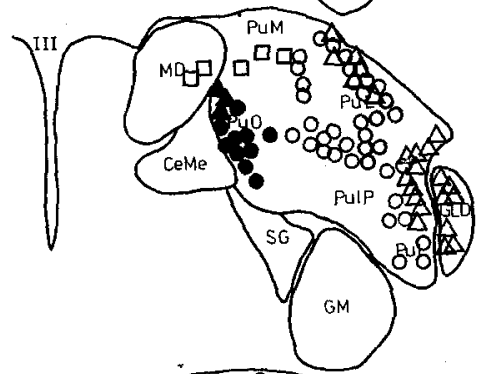

A 3

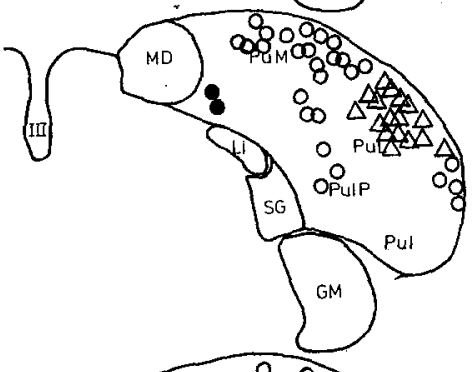

A 2.5

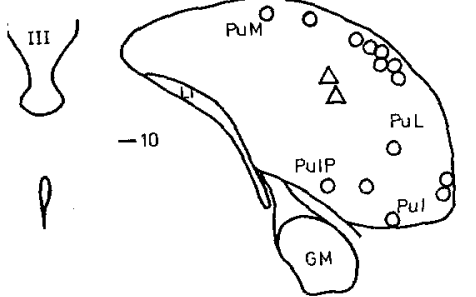

A 2
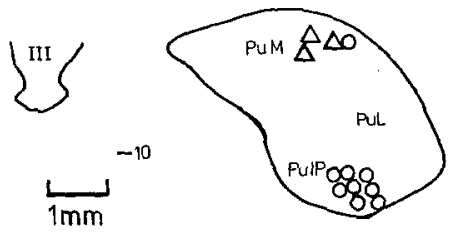

Fig. 6. Diagrammatic representation of the injections combined in gradient I (see text) and their respective thalamic labellings. Symbols of thalamic labellings correspond to the symbols used to mark the respective cortical injection sites. They represent the approximate density of thalamic labelling, but not the exact cell number rule, especially into VA but also into VL, LP, MD and the medial pulvinar. Using morphologic criteria alone it is indeed difficult if not impossible to draw a clear border between $\mathrm{CeL}, \mathrm{CeD}$, and the neighbouring nuclei. It may be possible that non-specific and specific projection neurons are intermingled in these borderline regions between the intralaminar and specific nuclei. VA seems to be a special case in that a direct continuity between labellings in VA and the intralaminar nuclei could be observed in all experiments, labelling both those regions (e.g. Figs. 3-5). Also the overlap between labelling fields from different cortical injections is clearly higher in both, the medial VA and the intralaminar nuclei than in the specific thalamic nuclei. This indicates that at least the medial part of VA can be considered as belonging to the intralaminar system (cf. Brysch et al. 1984). Macchi et al. (1984) suggest that except for the magnocellular neurons in VA, all other neurons of this nucleus should be ac- 


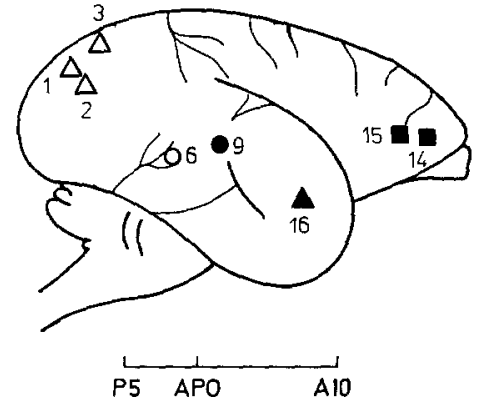

A 7

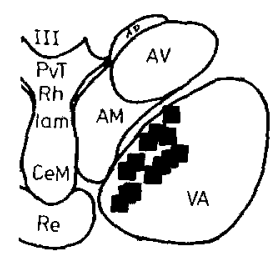

A 6.5

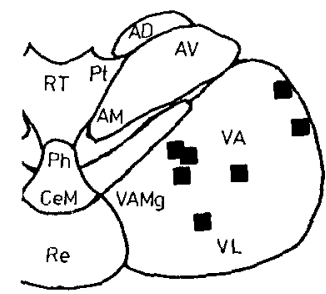

A 6

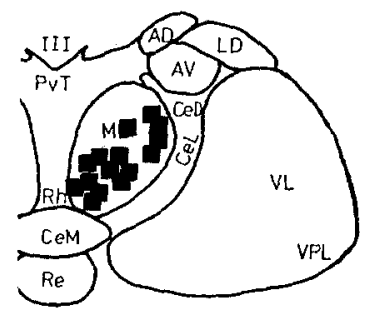

A 5.5
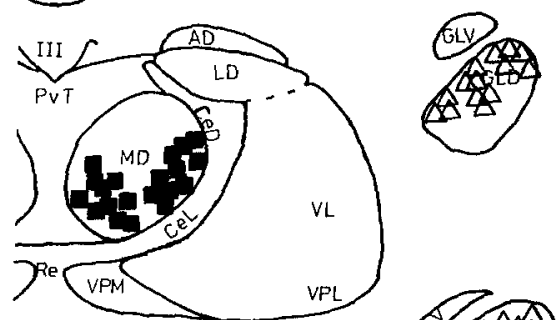

A 5

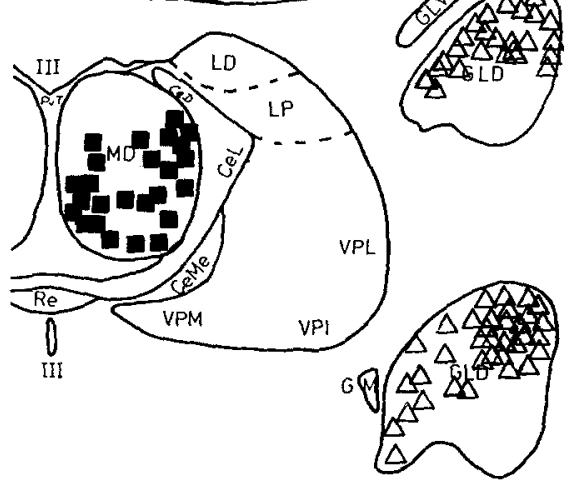

A 4.5

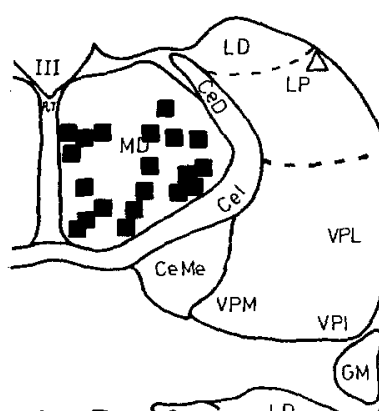

A 4

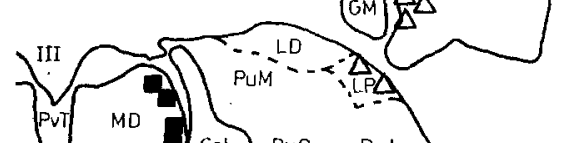

A 3.5

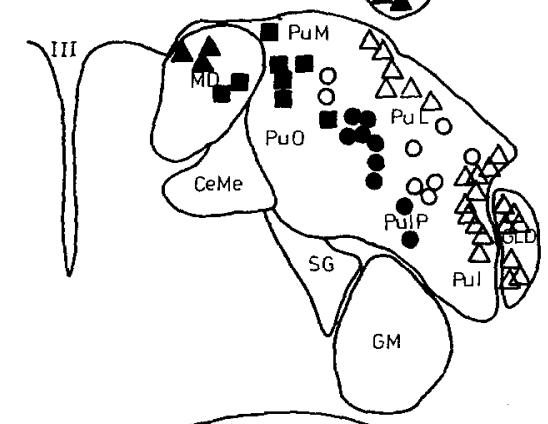

A 3

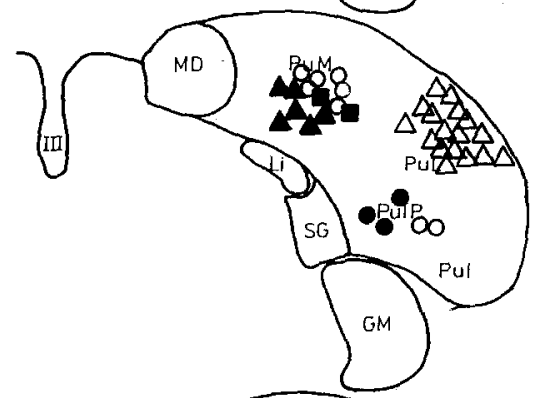

A 2.5
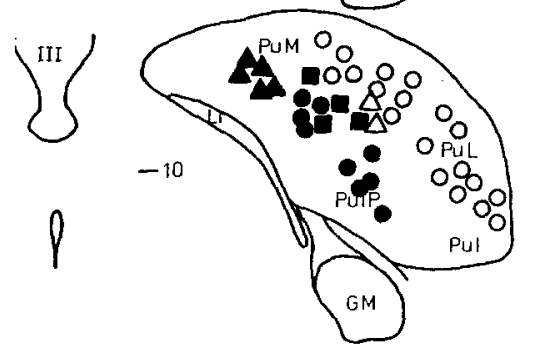

A 2

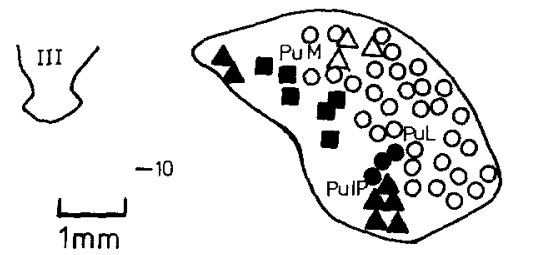

Fig. 7. Diagrammatic representation of the injections combined in gradient II (see text) and their respective thalamic labellings. Symbols of thalamic labellings correspond to the symbols used to mark the respective cortical injection sites, representing approximate density of labelled neurons counted to the intralaminar system. Jones (1985) regards even the magnocellular part of VA as part of it.

In Figs. 6-8 and 11, which consider only the projections from the specific thalamic nuclei and in which several experiments were combined, we have deliberately left out the intralaminar labelling zones because of the high degree of overlap within these nuclei. Labelling in VA was excluded, if clearly separate from the specific 


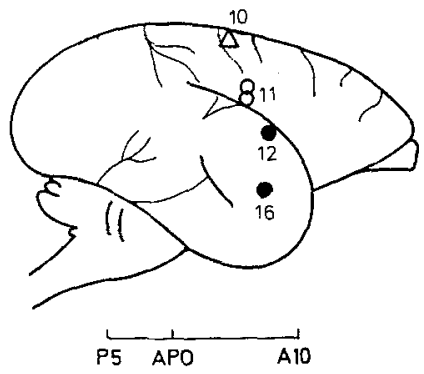

A 7

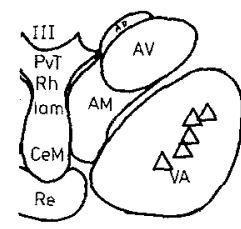

A 6.5

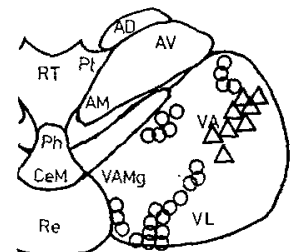

A 6

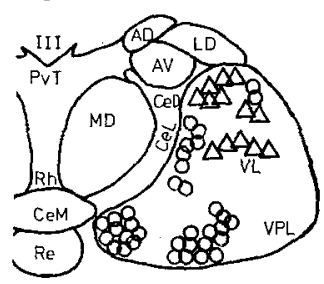

A 5.5
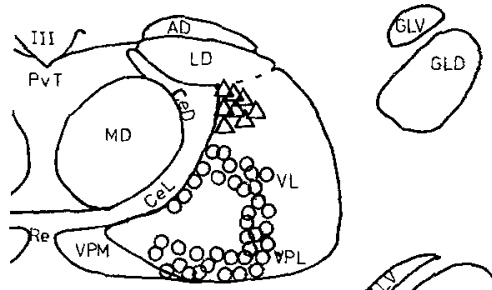

A 5

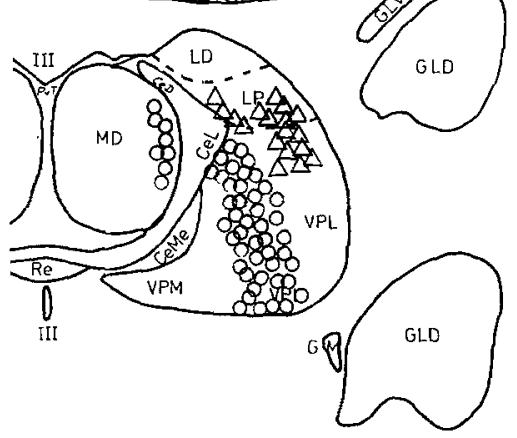

A 4.5

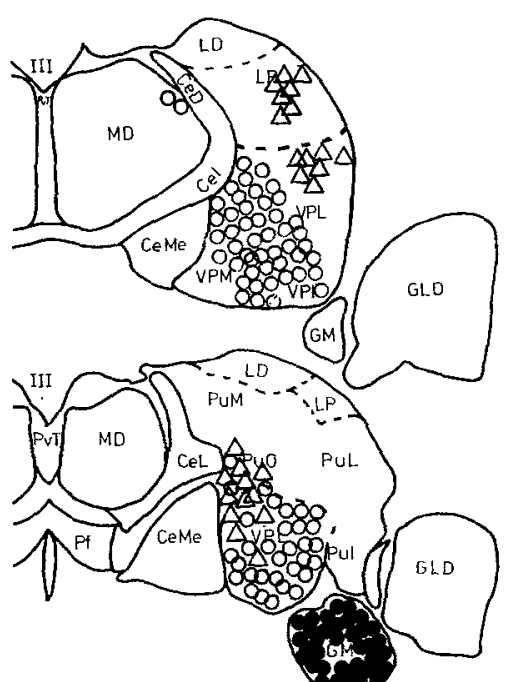

A 3.5

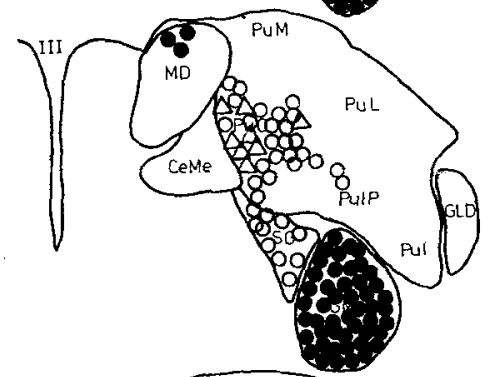

A 3

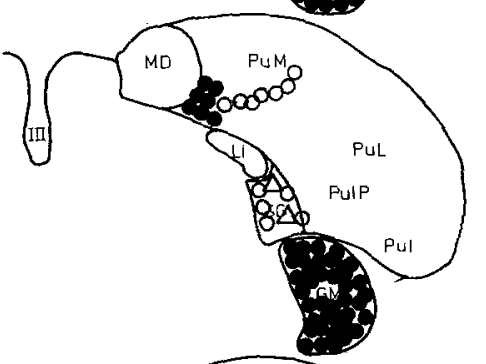

A 2.5

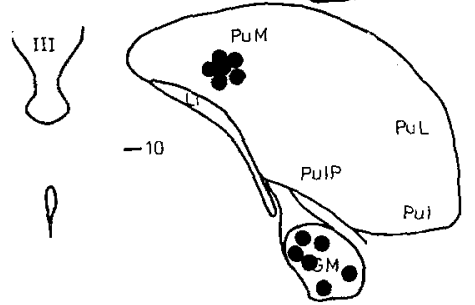

A 2

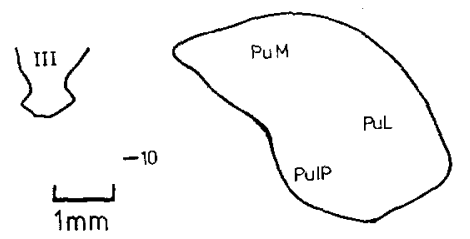

Fig. 8. Diagrammatic representation of the injections combined in gradient III (see text) and their respective thalamic labellings. Symbols of thalamic labellings correspond to the symbols used to mark the respective cortical injection sites. Approximate cell densities are shown projection zone and continuous with the intralaminar staining in $\mathrm{CeL}$ and/or $\mathrm{CeD}$, such as in experiment 6 (Fig. 5).

\section{Topological gradients in thalamus and cortex}

In the following we will combine sequences of cortical injections along vectors, which represent approximately continuous lines in the two-dimensional map of Fig. 1C:
I: The parasagittal caudo-rostral series of injections (Fig. 6), running dorsally along the interhemispheric fissure from caudal to rostral including injections $1 / 2 / 3$, $4 / 5,8 / 10,13$ and 14.

II: The lateral rostro-caudal vector (Fig. 7) running along the lateral periphery of the cortical convexity from the occipital cortex through the temporal lobe and ending in the prefrontal cortex. It includes injections $1 / 2 / 3$, $6,9,16$ and $14 / 15$.

III: The central medio-lateral series of injections 
crossing the Sylvian fissure, stretching from the parietal cortex through the parietal operculum to the infrasylvian cortex, including injections 10, 11, and 12b/16 (Fig. 8).

Vector $I$. The most caudal cortical injections considered here were experiments 1, 2 and 3 (open triangles in Fig. 6). They are combined into a single large injection zone. The cortical location of injections 1,2 and 3 is at the borderline between area 17 and 18 , but also reaches into area 19 (injection 3). Thalamic labelling was concentrated in the dorsal and medial segments of GLD and the most lateral segment of the pulvinar $(\mathrm{PuL})$. Intralaminar labelling (not shown) was concentrated between A 6.5 and A 5, extending with several labelled cells into the adjacent regions of VA, VL, nucleus lateralis dorsalis (LD) and LP.

Injections 4 and 5 are both located in the border zone between area 19 and 7 . Thalamic labelling (open circles in Fig. 6) occupies a zone in the latero-dorsal segments of LP, PuL and pulvinar medialis (PuM) from A 4.5 to A 2.5 and then stretches through the central part of the pulvinar to its ventro-lateral segment from A 3.5-2.0. Intralaminar labelling (not shown) was densest in $\mathrm{CeL}$ and $\mathrm{CeD}$ between $\mathrm{A} 6.5$ and 5.5 , but continued until $\mathrm{A} 4$ and extended with a few cells into VA, LD and PuM.

Injections 8 and 10 stained the parasagittal segments of the parietal and postcentral cortex (areas 7, 5 and $1 / 3$ ). Thalamic labelling (filled circles in Fig. 6) in both experiments was strongest in LP and also reached into VL, the caudal part of VPL and the antero-medial pulvinar. Intralaminar labelling (not shown) was confined to $\mathrm{CeL}$ and $\mathrm{CeD}$ in continuity with the labelling zones in LP and PuM.

Injection 13 was located in the transition zone between areas 4 and 6 . Thalamic labelling stretched from the lateral segment of VA at A 7.0 through the mediodorsal segment of VL towards the latero-ventral segment of $\mathrm{MD}$ (filled triangles in Fig. 6). A few labelled cells were scattered in PuM at A 3.5, just adjacent to MD. The labelling in all of these nuclei was continuous with the labelling in CeL (see Fig. 4).

In experiment 14, the prefrontal cortex (area 9) was injected. Thalamic labelling (open squares in Fig. 6) was densest in the medial segment of VA and in MD. Labelling in MD started more rostrally than in experiment 13, and reached into its caudal end with only a few cells. Few cells were also labelled in the rostral part of PuM, adjacent to MD. Some intralaminar neurons were labelled in the most anterior part of CeL, close to the VA and MD zones.

In summary, this caudo-rostral series of parasagittal cortical injections labelled a continuous thalamic zone, which starts in the most lateral part of the thalamus, the GLD, turns medio-dorsally into PuL and continues along the dorso-lateral surface of the pulvinar (including its most caudal end) through LP and the dorso-lateral part of VL and VA into MD.

Vector II. This rostro-caudal vector covers the lateral periphery of the hemisphere from the occipital through the temporal to the frontal lobe (Fig. 7). As the most caudal injection site we again combined experiments 1 , 2 and 3 in which the striate and prestriate cortex were injected. The field of labelled neurons in the specific thalamic projection nuclei is indicated by open triangles in Fig. 7.

In experiment 6, HRP was injected into the posterior part of the temporal association cortex $3 \mathrm{~mm}$ above the lateral edge of the temporal lobe involving areas 20 and 21. Labelled neurons (open circles in Fig. 7) were densely packed throughout the latero-dorsal half of the pulvinar, including its posterior pole. Intralaminar staining was concentrated in the anterior sections of $\mathrm{CeL}$ and $\mathrm{CeD}$ with considerable spread into the adjacent medial segment of VA (see Fig. 5).

In experiment 9 , the transition between the parietal (area 7) and the temporal association cortex (area 22) was injected. This relatively small injection labelled only few cells mainly in the basal segments of the posterior pulvinar (filled circles in Fig. 7). A few intralaminar cells were stained in $\mathrm{CeL} / \mathrm{CeD}$ at $\mathrm{A} 6$.

In experiment 16 the auditory and periauditory cortex were injected. Labelled thalamic cells (filled triangles in Fig. 7) were found in the anterior medial geniculate GM (plane A 4), in the medial segment of the pulvinar between A 3 and A 2 and a few scattered cells in MD (plane A 3.5).

The most rostral injections in this series were numbers 14 and 15 into the prefrontal cortex (areas 8 and 9). The thalamic labelling zone (filled squares in Fig. 7) stretched from the medial segment of VA through the latero-basal parts of MD and terminated caudally in the adjacent medial segment of the pulvinar with some cells lying as far posteriorly as A 2.5. This pulvinar zone is continuous with the labelling zone of injection 16 . In the non-specific nuclei, only the most anterior part of $\mathrm{CeL}$ was labelled.

In summary, this caudo-rostral cortical vector along the lateral circumference of the convexity corresponds to a thalamic labelling zone, which starts in GLD and stretches caudally through the latero-basal into the caudal pulvinar and GM. It then turns medially and anteriorly through $\mathrm{MD}$ and terminates in the medial VA. Note, that the thalamic projection zones to the lateral prefrontal and the rostro-lateral temporal regions touch and overlap in the rostro-medial pulvinar (see Discussion).

Vector III. This medio-lateral cortical vector stretches from the parietal cortex (injection 10) through the suprasylvian postcentral (injection 11) to the infrasylvian temporal cortex (injections $12 \mathrm{~b}$ and 16). Injection 10 stains both, the parasagittal part of area 5 and the adjacent postcentral cortex (area 1 and 3). The labelled thalamic region (open triangles in Fig. 8) stretches from the lateral segment of VA through VL into the laterodorsal part of LP and the dorsal part of VPL, ending in the most medial section of PuO.

Injection 11 is located in the suprasylvian, $12 \mathrm{~b}$ and 16 are in the infrasylvian cortex. Thalamic labelling from experiment 11 (open circles) reached from VA through 
the medial part of VL, and the medial and posterior VPL into the pulvinar ( $\mathrm{PuM}$ and $\mathrm{PuO}$ ).

Of experiment 12 we only included staining in GM (filled circles in Fig. 8) although injection 12 had affected both, the supra- and infrasylvian cortex (see Figs. 1C and 2). It therefore labelled cells in a thalamic region, which overlapped with labelling zones from injections 11 (ventro-basal complex and pulvinar, Fig. 3) and 16 (pulvinar and GM, filled circles in Fig. 8; see also Figs. 11C, D). In contrast to injection 16 however, it showed labelling in almost the entire GM.

In summary this central medio-lateral cortical stripe labelled neurons in the middle part of the thalamus beginning dorso-laterally and terminating medio-ventrally.

\section{Discussion}

\section{Topological principles of thalamo cortical projections}

In our experiments all thalamic labelling fields resulting from neighbouring cortical injections were adjacent and some of them overlapped to a certain extent. This is consistent with a topological organization of thalamocortical projections and confirms on a coarse scale what is known on the microscale in the various thalamo-cortical projection fields where the topological principle is known as somato-, retino- or tonotopy, respectively. On the other hand, thalamic projection zones to widely separated cortical areas in the temporo-parietal and frontal association cortex may contact each other (see e.g. injections 14 and $4 / 5$ in Fig. 6, plane A 3.5 and injections 6,16 and $14 / 15$ in Fig. 7, planes A 2-A 3.5).

When trying to extract a general topological principle of thalamo-cortical mapping, there is the problem that the thalamic cell groups projecting into a large circular cortical region as injected in our experiments are not spheres but elongated cones or shells, the long axes of which are oriented somewhat differently in different thalamic regions. As a result, progression along a cortical straight line does not correspond to a straight progression in the thalamus necessarily. This is obvious from Figs. $9 \mathrm{~A}$ and $9 \mathrm{~B}$, where we transferred the various thalamic projection fields of gradients I and III into a threedimensional model of the thalamus.

In spite of the sometimes tortuous course of the various projection zones a coherent scheme emerges from these drawings. Thalamic labelling zones resulting from the parasagittal row of cortical injections (gradient I) fill the dorso-lateral half of the anterior thalamus, but leave free the most dorsal region i.e. the nuclei LD and anterior ventralis (AV). These are the projection nuclei to the cingular cortex (not injected in our experiments) which is the mesial continuation of this cortical zone. In the caudal half of the thalamus much of the anterior pulvinar is labelled, as well as the GLD but the caudal pole of the thalamus shows relatively few labelled cells. (The caudal pole is not seen in the perspective drawing of Fig. 9, and the left block in Fig. 9A therefore gives the false impression as if labelling extends into the caudal pulvinar. A more precise picture is given in the original plots of Fig. 6.)
This caudal pole of the thalamus was labelled strongly by injections into the occipito-temporal and temporal cortex, combined in gradient II (see Fig. 7). This gradient, a semicircle round the lateral part of the cortex, results in a semicircular labelling in the thalamus, which starts in GLD, but then runs medially and backwards through the lateral into the caudal end of the pulvinar, labelling its lateral, inferior and posterior subdivisions. There it turns rostrally and runs through the medial part of the pulvinar (PuM) into $\mathrm{MD}$, which is covered from plane A 3.5 up to its anterior pole. The posterior thalamic region was difficult to draw in our three-dimensional sketch of the thalamus since the caudal pole of the pulvinar is filled with shells of labelling from all five groups of experiments, which obstruct each other in this perspective. The gradient is therefore not shown as a 3-D drawing, but its general appearance can well be extracted from Fig. 7. The ventral parts of the thalamus namely VPL, VL and the medio-ventral pulvinar as well as LP are labelled by the injections into the anterior-parietal, the perisylvian and the sensory-motor cortex, combined in gradient III (Fig. 9B).

\section{Concentric zones in the cortex and their respective thalamic projection zones}

In the following we regard the central cortical region (parietal, periinsular and sensory-motor cortex) as a core zone. Instead of straight lines of injections we consider approximately concentric zones, surrounding this central cortical region (Fig. 10A). Each zone has a segment in the frontal, the occipito-parietal and the temporal lobe. Arrows in Fig. 10A point from core zone 1 to the outmost zone 4 in each of these segments. Thalamic labellings from injections into these four cortical zones were combined into four corresponding thalamic zones (Fig. 10B). This lead to a coherent picture of thalamic labelling. In the thalamic coronal planes the arrows point from thalamic labelling zone 1 , which projects to the cortical core region to labelling zone 4 , which projects to the outmost cortical ring. The orientation of these arrows changes systematically from frontal to caudal. Figure $11 \mathrm{~A}-\mathrm{D}$ shows the plots for each group of injections and the respective thalamic labellings.

The cortical arrow pointing forward into the frontal cortex corresponds, in the anterior thalamus, to an arrow from ventro-lateral to dorso-medial (A6-A5 in Fig. 10B). This is in line with the findings of Kievit and Kuypers (1977) that the caudo-rostral progression in the frontal cortex corresponds to a latero-medial progression in the thalamus.

In the posterior thalamus, on the other hand, zones corresponding to cortical regions of increasing distance from the central core, are arranged in a slab like manner with the vectorial arrow pointing from medio-ventral to dorso-lateral. These zones are reminiscent of, and similar in orientation to the thalamic zones in the posterior thalamus of the cat resulting from a sequence of injections into the occipito-temporal and frontal cortex (Berson and Graybiel 1978; Creutzfeldt 1985). 

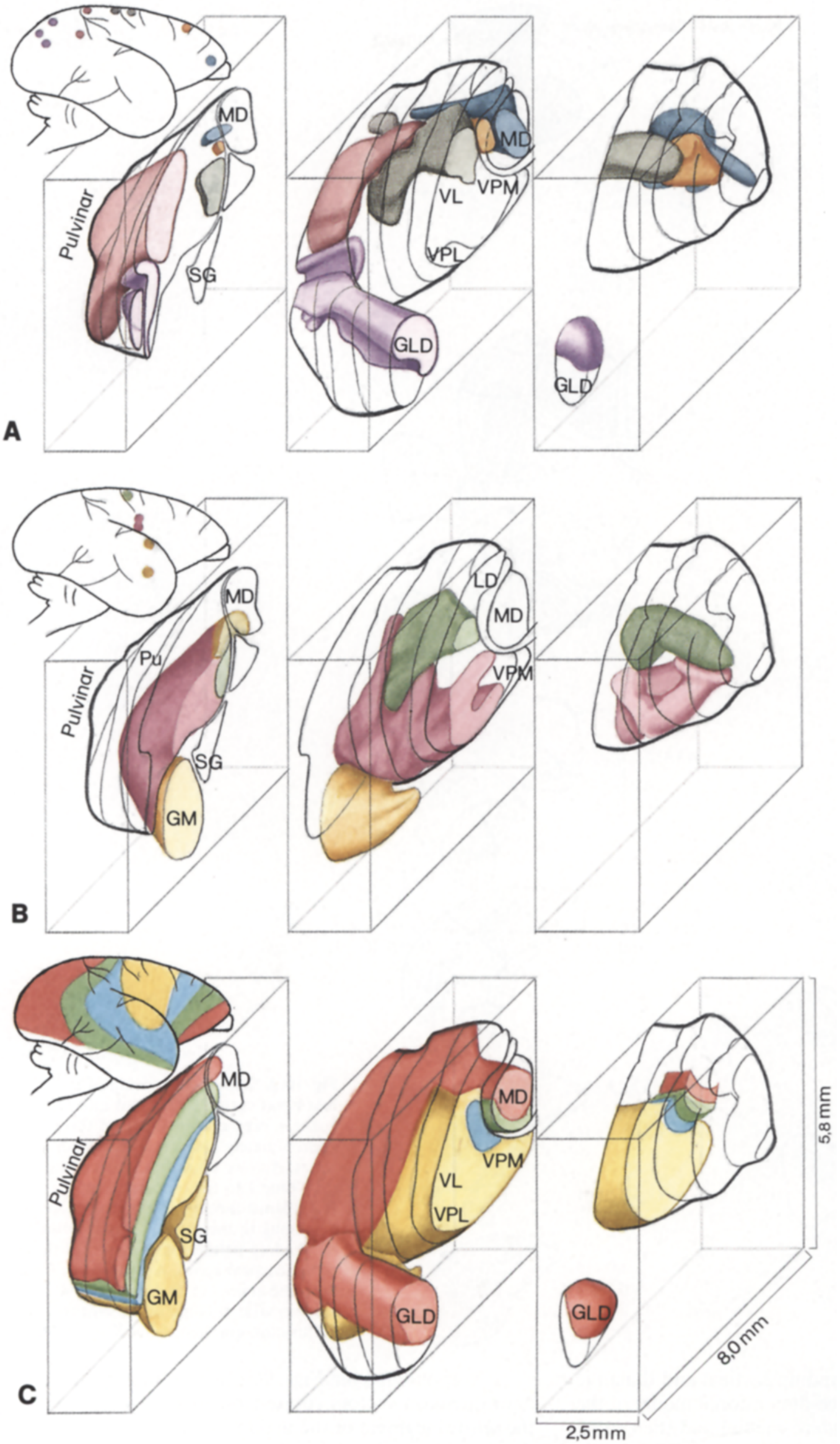

Fig. 9A-C. Three-dimensional reconstructions of thalamic labellings. A Gradient I.

B Gradient III. C The experiments grouped into four zones as in Fig. 10. The three-dimensional model of the marmoset thalamus was graphically reconstructed from schematic drawings of frontal sections as shown in Figs. $3-6$. The thalamic volume is divided into three blocks.

Divisions between the blocks are at planes A 3.5 and A 5.5. In addition a three-dimensional model was constructed from styrofoam to serve as a control for graphic reconstruction. Colours mark injection zones and their respective thalamic labelling fields. The caudal pole of the pulvinar is not visible in this perspective (see text) 


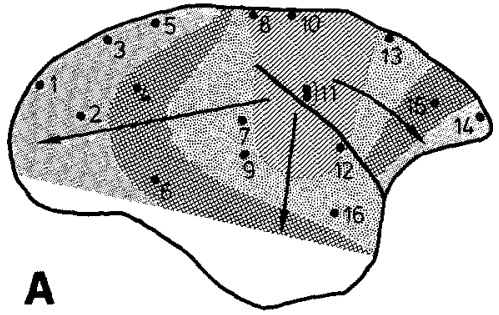

P5 APO A5 A10

10

A 7.5

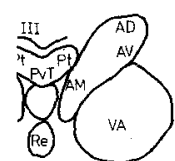

A 7

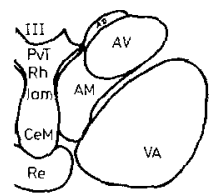

A 6.5

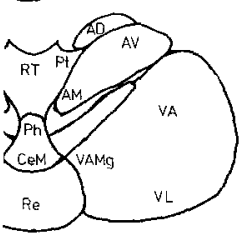

A 6
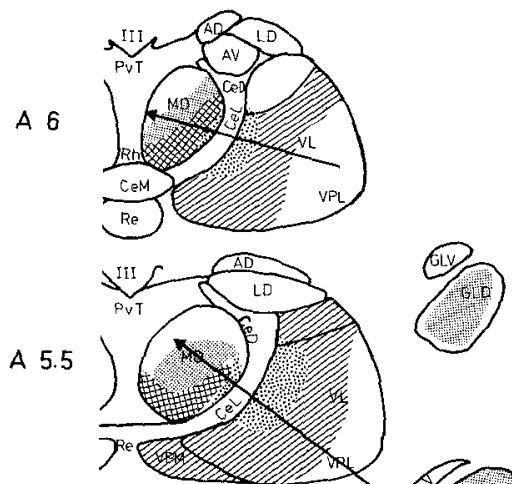

A 5

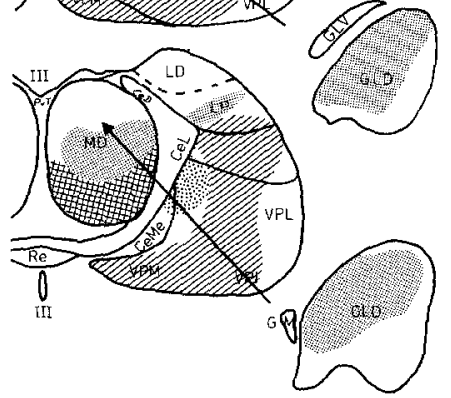

B

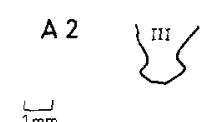

In Fig. 9C these corresponding cortical and thalamic zones are drawn into the three-dimensional model of the thalamus. Here the rostral latero-medial and the caudal medio-lateral thalamic progression zones can be clearly recognized.

A 4.5

A 4

A 3.5

A 3

A 2.5
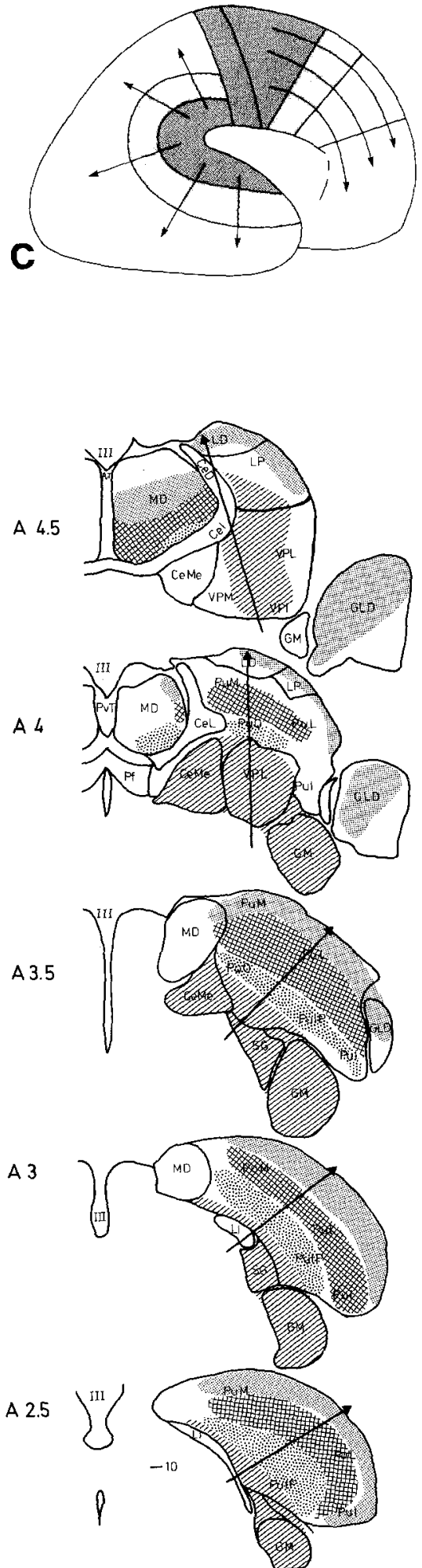

Fig. 10. A Schematic drawing of the marmoset cortex subdivided into four zones, a central core zone and three approximately concentric surrounding zones. Arrows point from the central core zone 1 to the outmost zone 4 . B Thalamic labellings from the injections belonging to the cortical zones shown in A. Shading patterns correspond to those of the cortical zones. Arrows point from thalamic zone 1 to thalamic zone 4 . C Schematic drawing of the cortical maturation gradient, from Kahle (1969)
It is apparent from Figs. 9C and 10B that the thalamic progression vectors rotate from rostral to caudal. In the central segment of the thalamus (A 3.5-A 4.5), where the ventral nuclei end and the pulvinar begins, the vector has its greatest rotation and the vectors in planes A 4 and 
A
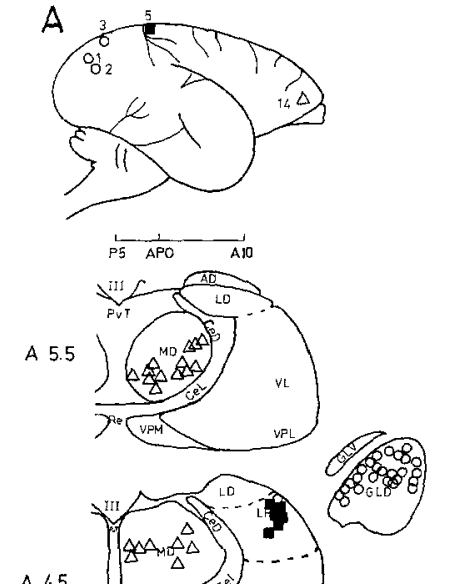

A 4.

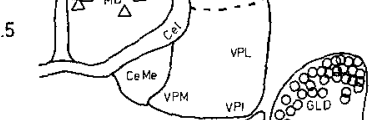

4
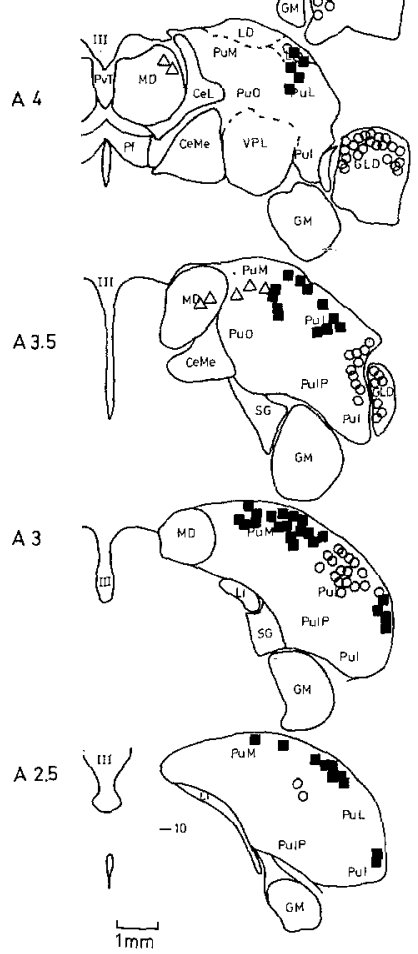

B
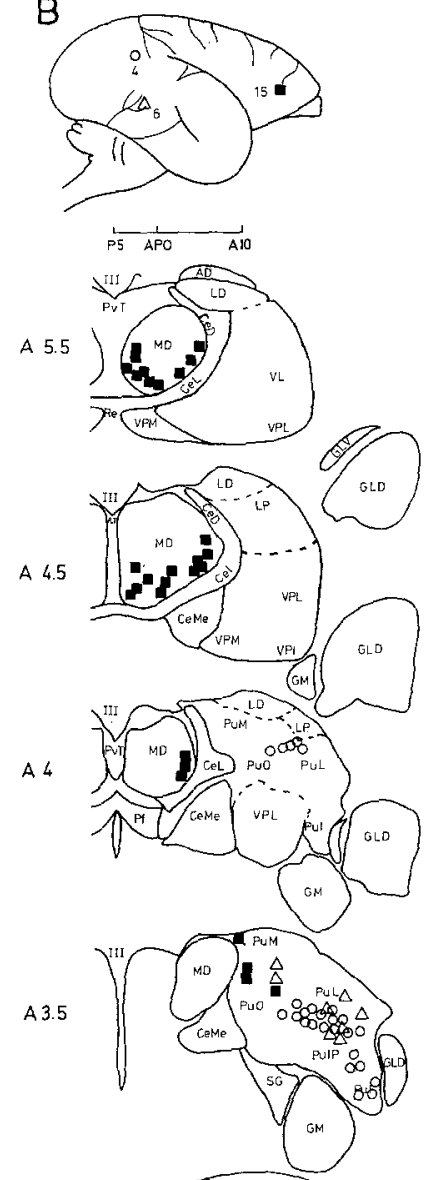

A 3

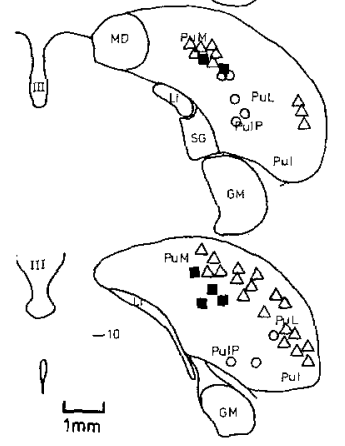

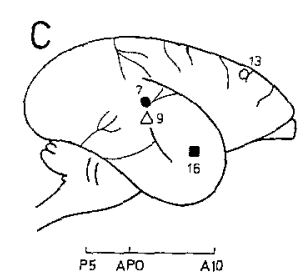
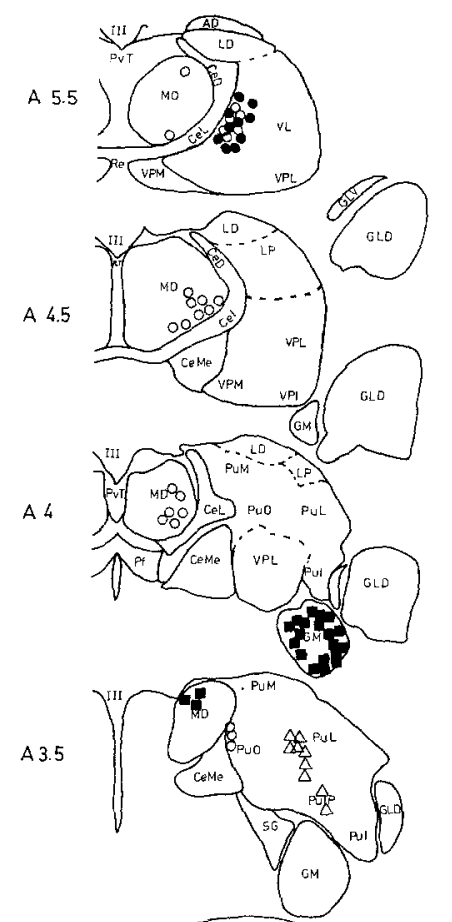

A 3

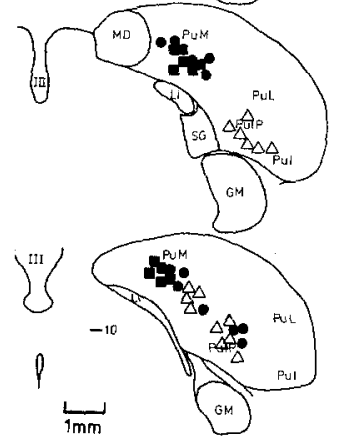

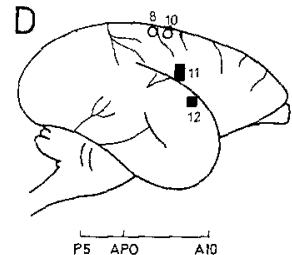
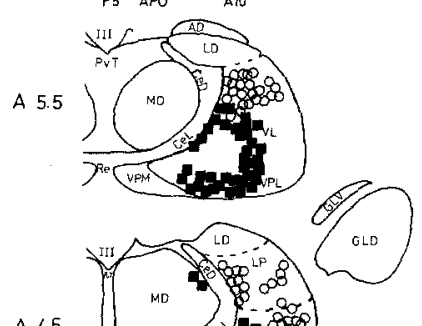

A 4.5
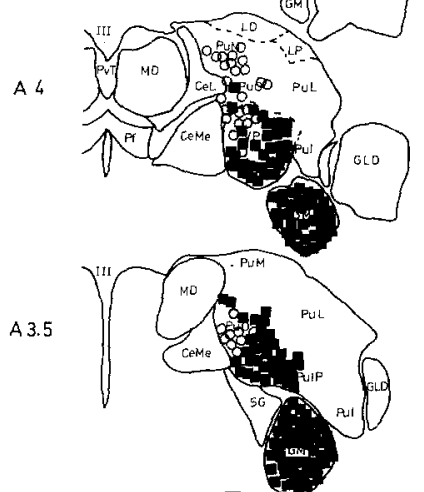

A 3

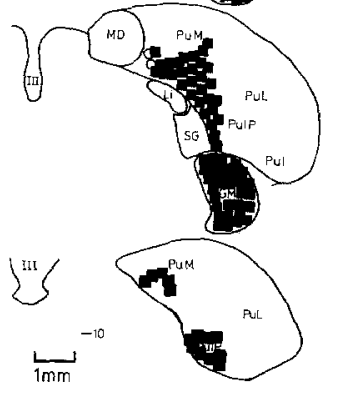

Fig. 11A-D. Diagrammatic illustration of thalamic labellings from four groups of injections. Each group represents one of the four zones as delineated in Fig. $10 \mathrm{~A}$. Symbols for thalamic labelling correspond to the symbols used to mark cortical injection sites

A 4.5 can only give an approximate direction but the predominant direction is between that in the frontal and the caudal thalamus.

This may be taken as an indication that the posterior thalamus is rotated relative to the anterior part. Such a rotation can in fact also be observed in single nuclei during ontogenetic development. Thus in the GLD of primates an initially latero-medial gradient (cells generated early being located laterally and late ones medially) turns into a ventro-dorsal one due to rotation of the primate GLD by about $110^{\circ}$ in the coronal plane (Rakic 1977). In analogy, the arrow in Fig. 10B rotates by about $120^{\circ}$ between plane A 6 and plane A 2 into the same direction as the one described by Rakic (clockwise in the left thalamus and anticlockwise in the right). A similar rotation can also be assumed for GM and the ventral posterior nucleus (VP), (see below).
Furthermore, a comparable latero-ventral rotation of GLD can also be recognized during phylogenetic development, in that the continuous GLD/pulvinar (or GLD/nucleus posterior/LP) zone which projects to areas 17 and the peristriate cortex, is located latero-dorsally in rodents (Hoehl-Abrahano and Creutzfeldt, in preparation), laterally in cats (Berson and Graybiel 1978) and latero-ventrally in the marmoset (open triangles in Fig. 6, section A 3.5). Similarly VP and GM are translocated further ventro-medially in primates as compared to lower mammals.

It is tempting to relate the cortical progression zones to the ontogenetic and phylogenetic development of the cortex. The time course of neuronal maturation in the cortex suggests that the oldest parts are the periinsular and central region and that during development of the cortex new material is added around this central core 
RELATIVE SIZE OF CERTAN THALAMIC NLCLE

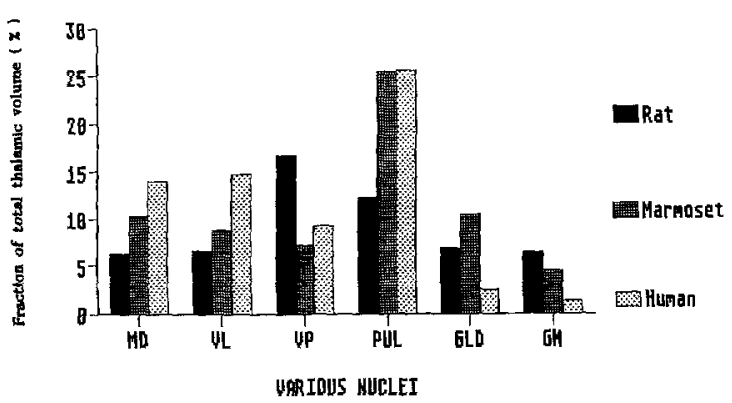

Fig. 12. Relative sizes of some thalamic nuclei in rat, marmoset, and man in percent of total thalamic volume. The volume of each nucleus was determined by measuring its area in frontal sections at constant intervals throughout the nucleus. The volume $\mathrm{V}$ of each nucleus was then calculated as follows: $V=a_{1-n} \times d$, where $\Sigma a_{1-n}$ is the sum of all individual areas of a nucleus in the various frontal planes, $d$ is the distance between two frontal planes. The measurements were done on Photomicrographs of the following atlases: Paxinos and Watson (1982) for the rat thalamus, Stephan et al. (1980) for the marmoset thalamus and Schaltenbrand and Bailey (1959) for the human thalamus. The atlas of Schaltenbrand and Bailey uses the classification and nomenclature of thalamic nuclei, developed by Hassler. The nomenclature used in this atlas was equated to the nomenclature used by Stephan et al. (1980) according to Duus (1983). Note that in the rat thalamus there is no pulvinar. In this figure we compare the size of the rat nucleus posterior to the primate pulvinar. This should not imply the grade of homology, however. It has to be emphasized that the graph does not consider cell density which, for example in the parvocellular layers of the primate GLD, is much higher than in other nuclei of the thalamus

(Kahle 1969), see Fig. 10C. For the additions in the frontal, temporal and parietal association cortex this is consistent with the sequential myelination of cortical areas (Flechsig 1920). It is not so for the occipital cortex. Flechsig's studies suggest that myelination in the occipital cortex follows a different topographic pattern, beginning in area 17. Myelination and birthdate of cortical neurons however are not necessarily correlated.

Rakic (1976; Rakic et al. 1986) pointed out that neurons in area 19 are on average generated earlier than those of area 18 and these in turn are older than those in area 17 as determined by the ${ }^{3} \mathrm{H}$-thymidine method. This is consistent with Kahle's maturation gradients. Rakic's (1976) study does not cover areas 5 and 7, which lie between the postcentral region and area 19. To our knowledge the neuronal generation time of this part of the association cortex relative to the visual cortical areas 17-19 has not been studied with the ${ }^{3} \mathrm{H}$-thymidine method in primates.

If we assume that neurons in thalamic zones are generated along the same temporal sequence as those in the cortical zones to which they project, both the cortical and thalamic vectors should be related to developmental vectors. The arrowheads in Fig. $10 \mathrm{~A}$ and $\mathrm{B}$ would then point to the newest additions of neurons in the thalamus and cortex and the tails would correspond to the older parts. This would mean that new material in the rostral thalamus is added medially. This is consistent with de-
A
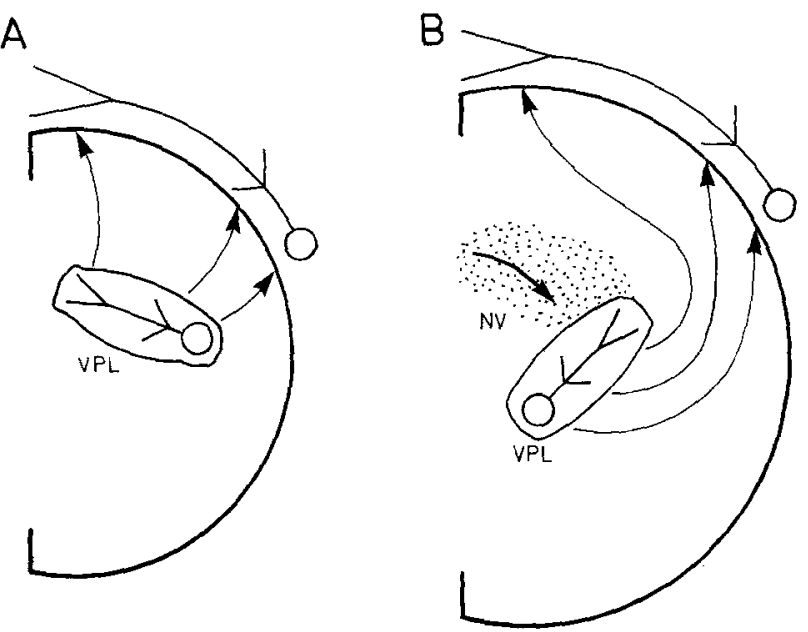

Fig. 13A, B. Cartoon showing the development of the inversion of somato-topy in the somato-sensory cortex and in VPL. In this model it is assumed that at an early developmental stage $(\mathbf{A})$ the thalamo-cortical projections are essentially radial. At a later stage, newly generated thalamic volume (NV) in the dorso-medial thalamus pushes VPL laterally and ventrally, but the connections remain towed to their original target regions (B). It should be noted that this scheme refers to the development of the thalamo-cortical topology only. The somatotopic organisation is projected on this and is the consequence of the arrangement of somatic afferents into the thalamus, which is still absent during the earliest stages of thalamic and cortical development

velopmental studies on cell generation in the thalamus of mice and rats, which indicate that neuronal generation takes place in a latero-medial direction, i.e. cells in the lateral thalamus are generated first and those in the medial thalamic region last (Angevine 1970; Altman and Bayer 1979; Schlingensiepen et al. 1989). This corresponds to the direction of our topological vector in the anterior half of the thalamus.

Phylogenetically the posterior thalamus (pulvinar/ LP-complex as compared to the nucleus posterior/LPcomplex) increases in relative size from rodents to primates as do the projection nuclei to the frontal and prefrontal cortex MD and VL (see Fig. 12). GM (and in humans even GLD) in contrast decreases in relative size due to the relative increase of other thalamic nuclei.

The more complicated vector in the central thalamus and its rotation towards more caudal parts of the thalamus could result from the fact that new material generated medially pushes older material laterally and ventrally, resulting in an apparent rotation during development. In the caudal thalamus (pulvinar) new material should thus be added as caps around the dorsocaudal surface. The ventro-basal complex as well as the medial and lateral geniculate bodies are pushed ventrally by medio-dorsally added new material in the anterior thalamus and at the same time rotated. Such a rotation could explain the reversal of somato-topic representation in the ventro-basal complex with respect to that in the sensory-motor cortex, as sketched in Fig. 13. Also the tonotopic arrangement in GM is rotated relative to that in the auditory cortex as shown in guinea pig and cat (Redies et al. 1989; Brandner and Redies, 1990) and 
the visuo-topic maps in GLD and area 17 are rotated by about $90^{\circ}$ relative to each other.

Based on our findings and the above considerations we propose that the topology of thalamo-cortical projections is a consequence of the temporal sequence of development in both structures. This would not only give a clue to the topological transformations of thalamocortical projections but also would explain the fact that thalamic zones projecting to the parietal, occipitotemporal and frontal association cortex are adjacent or overlapping in the contact region between the caudal tail of MD and the adjacent part of PuM (see injections 5 and 14 in Fig. 11A; 4, 6 and 15 in Fig. 11B and 7, 9, 13 and 16 in Fig. $11 \mathrm{C}$ ). In all these cases the respective cortical projection zones are far apart from each other, but they can all be considered as later additions to the "central core" region, receiving input from the latest thalamic additions. The model furthermore explains the inversion of functional organotopic maps from thalamus to cortex.

This proposal should be considered as a heuristic model, which could explain many riddles of the topology of thalamo-cortical organisation and it may be applicable also to other topological maps in the central nervous system. Temporal sequences during development could provide a basic scaffolding for later differentiation of the respective maps.

Acknowledgements. The authors gratefully acknowledge the help of Dr. Sven Ebbesson during the first experiments, and the continuous advice and assistance of Susanne Lausmann throughout this study.

\section{References}

Altman J, Bayer S (1979) Development of the diencephalon in the rat: thymidine-radiographic observations on the internuclear and intranuclear gradients in the thalamus. J Comp Neurol 188:473-500

Angevine JB (1970) Time of neuron origin in the diencephalon of the mouse: an autoradiographic study. J Comp Neurol $139: 129-188$

Berson DM, Graybiel AM (1978) Parallel thalamic zones in the LP-pulvinar complex of the cat identified by their afferent and efferent connections. Brain Res 147:139-148

Brandner S, Redies H (1990) The projection from medial geniculate to field A I in cat: organisation in the isofrequenzy dimension. J Neurosci 10:50-61

Brodmann K (1909) Vergleichende Lokalisationslehre der Großhirnrinde. Barth, Leipzig (reprinted 1985)

Brysch I, Brysch W, Creutzfeldt OD, Hayes NL, Schlingensiepen K-H (1984) The second, intralaminar thalamo-cortical projection system. Anat Embryol 169:111-118

Brysch W (1987) Thalamocortikale Projektionen beim Marmoset. Dissertation. Göttingen
Creutzfeldt OD (1985) Comparative aspects of representation in the visual system. Exp Brain Res Suppl 11:53-81

Duus P (1983) Neurologisch-topische Diagnostik. Thieme, Stuttgart

Flechsig P (1920) Anatomic des menschlichen Gehirns und Rückenmarks auf myelogenetischer Grundlage. Thieme, Leipzig

Goldman-Rakic P, Porrino L (1985) The primate mediodorsal (MD) nucleus and its projection to the frontal lobe. J Comp Neurol 242:535-560

Hassler R (1950) Die Anatomie des Thalamus. Arch Psychiatr Nervenkr 184:249-256

Hassler R, Mundinger F, Riechert T (1979) Stereotaxis in Parkinson syndrome. Springer, Berlin Heidelberg New York

Hirai T, Jones EG (1989) A new parcellation of the human thalamus on the basis of histochemical staining. Brain Res Rev $14: 1-34$

Jones EG (1974) The anatomy of extrageniculostriate visual mechanisms. In: Schmitt FO, Worden FB (eds) The neurosciences: third study program. MIT Press, Cambridge Mass, pp 215--227

Jones EG (1985) The thalamus. Plenum, New York

Kahle W (1969) Die Entwicklung der menschlichen Großhirnhemisphäre. Springer, Berlin Heidelberg New York

Kievit J, Kuypers H (1977) Organisation of the thalamo-cortical connections in the frontal lobe in the rhesus monkey. Exp Brain Res 29:299-322

Macchi G, Bentivoglio M, Molinari M, Minciacchi D (1984) The thalamo-caudate versus thalamo-cortical projections as studied in the cat with fluorescent retrograde double labelling. Exp Brain Res 54:225-239

Malone E (1910) Über die Kerne des menschlichen Diencephalon. Verhandl Königl Akad Wiss, Berlin

Mesulam MM (1982) Tracing neural connections with horseradishperoxidase. $\mathrm{J}$ Wiley and Sons, Chichester

Paxinos G, Watson C (1982) The rat brain in stereotaxic coordinates. Academic Press, Sydney

Rakic P (1976) Differences in the time of origin and in eventual distribution of neurons in areas 17 and 18 of the visual cortex in rhesus monkey. Exp Brain Res Suppl 1:244-248

Rakic P (1977) Genesis of the dorsal lateral geniculate nucleus in the rhesus monkey: site and time of origin, kinetics of proliferation, routes of migration and pattern of distribution of neurons. J Comp Neurol 176:23-52

Rakic P, Bourgeois J-P, Eckenhoff MF, Zecevi N, Goldman-Rakic PS (1986) Concurrent overproduction of synapses in diverse regions of the primate cerebral cortex. Science 232:232-235

Redies H, Brandner S, Creutzfeldt OD (1989) Anatomy of the auditory thalamocortical system of the guinea pig. J Comp Neurol 282:489-511

Revishchin AV, Garey LJ (1990) The thalamic projection to the sensory neocortex of the porpoise, Phocoena phocoena. J Anat (in press)

Schlingensiepen R, Creutzfeldt OD, Schlingensiepen K-H (1989) Development of the thalamus in the mouse - an autoradiographic study with ${ }^{3} \mathrm{H}$-thymidine. Eur J Neurosci Suppl 2:52

Schaltenbrand G, Bailey P (1959) Stereotaxis with an atlas of the human brain. Thieme, Stuttgart

Stephan H, Baron G, Schwerdtfeger WK (1980) The brain of the common marmoset (Callithrix jacchus): a stereotactic atlas. Springer, Berlin Heidelberg New York

Vogt C (1909) La myeloarchitecture du thalamus du cercopithèque. J Psychol Neurol 12:285-324

Walker AE (1938) The primate thalamus. Chicago Press, Chicago 